Zwischen Verfassung und Präsidentenwillen. Der Umbau des Justizsystems in Polen

Bogna Baczyńska, Universität Stettin

DOKUMENTATION

Plenardebatte im Europäischen Parlament zur Rechtsstaatlichkeit in Polen,

19. Oktober 2021 (Auszüge)

UMFRAGEN

Bewertungen der Tätigkeit der Justiz durch die polnische Bevölkerung Einstellungen der polnischen Bevölkerung zur EU

$$
\begin{aligned}
& \text { Deutsches } \\
& \text { Polen-Institut }
\end{aligned}
$$




\section{Zwischen Verfassung und Präsidentenwillen. Der Umbau des Justizsystems in Polen}

Bogna Baczyńska, Universität Stettin

DOI: $10.31205 /$ PA.283.01

\section{Zusammenfassung}

Die Reformen des polnischen Justizsystems, welche die Regierung von Recht und Gerechtigkeit (Prawo i Sprawiedliwość-PiS) seit 2015 durchführt, tragen nicht nur zu einer möglicherweise fehlerbehafteten Rechtsprechung bei, sie fördern auch das Misstrauen in die polnische Rechtsstaatlichkeit und führen zu Konflikten im internationalen Bereich. Soeben hat der Gerichtshof der Europäischen Union (EuGH) Polen zu einer Strafzahlung verurteilt, da die Regierung die 2018 eingeführte Disziplinarkammer (Izba Dyscyplinarna) am, oder besser gesagt, über dem Obersten Gericht (Sąd Najwyższy) noch nicht aufgelöst hat. Die Veränderungen im Rechtssystem reichen tief und stellen Polens Zugehörigkeit zur europäischen Rechtsgemeinschaft infrage. Das Ziel der Regierung ist es, durch die Reform Justizpersonal, vor allem Richter, auszutauschen, um die Judikative als eine unabhängige Macht auszuschalten. Somit setzen die wesentlichen Maßnahmen - die Schaffung eines regierungskonformen (aber verfassungswidrigen) Landesjustizrates (Krajowa Rada Sadownictwa - KRS), die Disziplinarkammer am Obersten Gericht und die Verabschiedung des »Maulkorbgesetzes« - auf die Abschaffung der richterlichen Unabhängigkeit durch Beförderung politisch opportuner Richter oder Personen mit fehlenden Qualifikationen in Richterpositionen. Auf der anderen Seite bestraft die Regierung Richter, Staatsanwälte, Anwälte oder Rechtsberater, wenn sie ihren verfassungsmäßigen Pflichten nachgehen und sich der politischen Willkür im Justizbereich widersetzen.

\section{Einführung}

Als ich mich an diesen Artikel setzte, begann gerade die zweite Szene des neuesten Aktes im polnischen Justizdrama. In der ersten Szene des Aktes, am 7. Oktober 2021, hatte das Verfassungstribunal (Trybunat Konstytucyjny TK) unter der Ersten Präsidentin Julia Przyłębska - das heißt die Attrappe eines echten, rechtsstaatlichen Verfassungsgerichtes - ein neues Urteil gesprochen. Das "Gericht des letzten Wortes" stellte fest, dass das EURecht im Widerspruch zur polnischen Verfassung steht, auch wenn eine Kollision zurzeit nicht stattfindet. Es stellte weiter fest, dass die polnischen Gesetze über den europäischen Verträgen stehen, obwohl die Verfassung der Republik Polen deutlich den Vorrang internationaler Verträge benennt, sollten sie sich im Konflikt mit den Gesetzesvorschriften befinden. Das Verfassungstribunal spricht sich außerdem das Recht zu, die Urteile des Gerichtshofes der Europäischen Union (EuGH) in Luxemburg zu kontrollieren, auch wenn dies im Widerspruch nicht nur zu den EU-Verträgen, sondern auch zur polnischen Verfassung steht. Es kündigte Maßnahmen von Amts wegen an, um die Urteile des EuGH zu kontrollieren und sie aus dem Rechtsverkehr zu ziehen, obgleich es nur auf Antrag tätig werden kann und »die Urteile des EuGH aus dem Rechtsverkehr zu ziehen" - was immer das bedeuten mag - nicht in seinem Kompetenzbereich liegt. Kurz gesagt, es hat ein Urteil nicht auf der Basis des geltenden Rechtes, sondern auf Grundlage parteipolitischer und politischer Normen gesprochen. Zum Trost wies es darauf hin, dass solcherart Urteile vorher bereits in anderen Ländern gefällt wurden (u. a. in Deutschland und Frankreich) und dass bestimmte EU-Mitgliedsländer und ihre Verfassungsgerichte die Auffassung Polens teilen - was allerdings nicht wahr ist. Das Verfassungstribunal scheint in hervorragender Form zu sein, denn der Bruch der Verfassung, des EU-Rechtes und der internationalen Verpflichtungen gelingt ihm ohne Mühe.

Wie reagierte die polnische Gesellschaft darauf? Diese Antwort nun ist ernsthaft gemeint, denn schließlich berührt das Urteil des Verfassungstribunals auch eines der wichtigsten Persönlichkeitsrechte, das Recht auf ein faires, transparentes Gerichtsverfahren ohne unbegründete Verzögerungen. Der Hintergrund des ganzen Konfliktes ist, dass in den polnischen Gerichten Personen eingesetzt sind, welche die für Richter geltenden formalen Anforderungen nicht erfüllen. Auf dieses usurpatorisch-rechtlose Vorgehen des Verfassungstribunals reagierte die polnische Zivilgesellschaft am 10. Oktober 2021 mit Demonstrationen in den großen Städten. Die größte Demonstration mit 80.000 bis 100.000 Teilnehmern fand in Warschau statt. Sie protestierten gegen das Urteil des Verfassungstribunals und die Zerstörung der Judikative, gegen die Verletzung der Menschen- und Bürgerrechte sowie gegen den Prozess, Polen aus der Europäischen Union hinauszuführen. Die Demonstrationsteilnehmer unterstrichen mit skandierten, gesungenen oder geschriebenen Slogans, dass das zivilgesellschaftliche Polen nicht aus der EU austreten 
will und sich nicht mit dem Bruch der Verfassung und internationaler Verträge, darunter auch der europäischen Verträge, einverstanden erklärt. Die Mitgliedschaft Polens in der Europäischen Union erkannten sie als wichtigstes internationales Bündnis mit Schlüsselbedeutung an. Polen ist und bleibt Teil der EU, es ist (weitestgehend) geistig in der Kultur und dem politischen Denken des Westens verwurzelt.

Die zweite Szene des aktuellen Justizdrama-Aktes begann am 27. Oktober 2021. An diesem Tag belegte der Gerichtshof der Europäischen Union Polen mit einer Strafe in Höhe von einer Million Euro täglich, weil es die Disziplinarkammer (Izba Dyscyplinarna) am Obersten Gericht (Sąd Najwyższy) nicht ausgesetzt hat. Entgegen ihrer Bezeichnung dient die Kammer, die die Regierung von Recht und Gerechtigkeit (Prawo i Sprawiedliwość - PiS) einberufen hat, nicht der Disziplinierung, sondern der Einschüchterung von Richtern. Die Verpflichtung, sie aufzuheben, hatte der EuGH Polen bereits im Juli angetragen. Nur einen Tag nach der einstweiligen Verfügung verkündete der Gerichtshof, dass die Disziplinarkammer nicht mit dem EU-Recht vereinbar ist. Das bedeutete nicht nur, dass ihre Tätigkeit ausgesetzt, sondern dass sie aufgelöst werden muss, des Weiteren dass eine neue Gesetzgebung notwendig ist, um ein neues, rechtsstaatliches Organ zu schaffen. Leider hat Polen nicht nur nichts unternommen, sondern hat die Disziplinarkammer auch ostentativ ihre Tätigkeit fortgesetzt. Zu der genannten Strafe von einer Million Euro kommen außerdem 500.000 Euro Zwangsgeld täglich, weil die Tätigkeit des Bergwerkes Turów (Südwestpolen) nicht ausgesetzt wurde, was Tschechien beantragt hatte. 1,5 Millionen Euro Strafe pro Tag ist eine Summe, die sich der durchschnittliche Pole nicht vorstellen kann und die zeigt, dass die Geduld der EU fast am Ende ist. Gleichzeitig ist sich ein beträchtlicher Teil der Gesellschaft in Polen bewusst, dass die Zwangsgelder nicht unvermittelt verhängt wurden, sondern dass ihnen Berichte, Stellungnahmen, Appelle und Gutachten der EU vorangingen und die EU von ihren Mitgliedsstaaten unterstützt wurde und wird. Auch die Vereinigten Staaten von Amerika unter ihrem Präsidenten Joe Biden signalisieren deutlich, dass sie die neue Qualität der polnischen Politik nicht gutheißen, die ein Abdriften vom demokratischen Pfad bedeutet. Aber reicht das, um die gegenwärtige Entwicklung zu bremsen und wie konnte es überhaupt dazu kommen? Auf die erste Frage gibt es zurzeit keine Antwort. Auf die zweite versuche ich zu antworten, indem ich die wichtigsten Etappen des Konfliktes nachzeichne.

\section{Die Anfänge}

Der Sieg der PiS bei den Wahlen im Jahr 2015 (Präsident und Parlament) wurde von einem beträchtlichen
Teil der polnischen Gesellschaft begeistert aufgenommen. Nicht nur die Kernwählerschaft der PiS freute sich, sondern auch ein großer Teil der Gesellschaft, der vorher für die Bürgerplattform (Platforma Obywatelska $P O)$ oder eine andere Partei gestimmt hatte, hatte sich nun umentschieden. Eine weitere Unterstützergruppe waren die Wechselwähler; diese Gruppe ist in Polen größer als in den westlichen Ländern. Die PiS zog die Wähler mit ihrer energiegeladenen Rhetorik an, mit dem Versprechen, sich in der Außenpolitik und in europäischen Fragen "von den Knien zu erheben", und mit der Zusage von Sozialtransfers in einem Ausmaß, das es in Polen bisher noch nicht gegeben hatte. Letztere hat das Herz vieler Wähler geöffnet. Zweifel, ob Polen wirtschaftlich in der Lage ist, das Kindergeld-Vorzeigeprojekt " $500+« a b$ dem zweiten Kind zu schultern, zerstreute die damalige Ministerpräsidentin Beata Szydło mit der Beteuerung, dass der Wirtschaftsplan der PiS hervorragend sei, und bekräftigte dies mit der Feststellung, »es reicht aus, nicht zu klauen«, womit sie suggerierte, dass Diebstahl eine Spezialität der $P O$-Vorgängerregierung war. Der Wahlkampfslogan der PiS »Polen im Ruin« wurde sehr populär, auch wenn nicht einmal die überzeugten PiS-Anhänger diesen Ruin so richtig erkennen konnten. Die Europäische Union wurde als "Brüsseler» Bürokratie dargestellt, das heißt weit weg, fremd und im Grunde ein entbehrliches Gebilde. Anti-EU-Auftritte wurden als Ausdruck des Patriotismus behandelt. Diese fragwürdige Botschaft wurde mit Erzählungen über die vermeintliche moralische Zersetzung des Westens und die Überlegenheit des polnischen Katholizismus gewürzt; dieser sei jedwedem anderen Katholizismus sowie anderen Bekenntnissen überlegen. Dass die polnische katholische Kirche Papst Franziskus gegenüber taub war und immer noch ist, ebenso wie gegenüber den Problemen der Gegenwart und dem Thema Pädophilie und sexuelle Belästigung, und dass sie stattdessen mehr damit beschäftigt war (und immer noch ist), irdische Güter anzuhäufen und Politik zu betreiben, blieb eine kleine Nuance.

Allerdings waren aufmerksame Beobachter bereits von den ersten Taten der PiS nach ihrer Machtübernahme beunruhigt. Der neue, der PiS zugehörige Präsident Andrzej Duda sprach im Verlauf eines Korruptionsprozesses vier Angeklagte frei und überantwortete ihnen gleich nach dem umstrittenen Freispruch hohe und höchste Ämter in Ministerien - u. a. das des Ministers und Vizeministers für die Geheimdienste. Schockierend ist nicht allein die Tatsache, dass sie Ämter in so sensiblen Bereichen staatlichen Handelns übertragen bekamen, sondern auch, dass der Präsident sie gemäß dem in Polen geltenden Recht nicht begnadigen durfte. Der Grund: Nachdem das Gericht erster Instanz sie in seinem Urteil der Korruption für schuldig befun- 
den hatte, legten sie beim Gericht zweiter Instanz Berufung ein. Sie waren im juristischen Sinne immer noch unschuldig, denn das Urteil der ersten Instanz war noch nicht rechtskräftig. Die Begnadigung formal unschuldiger Personen rief einen Schock hervor, den die Worte Dudas, er habe beschlossen, dem Gericht "auszuhelfen", noch verstärkten. Dass das Gericht in Warschau den Prozess nicht fortsetzte, trotz des offenkundigen Mangels an Legalität und Gültigkeit dieser Begnadigungen, war ein erstes Symptom dafür, dass die Normen des allgemein geltenden Rechtes vor dem Willen der Exekutive zurückwichen. Der Freispruch von »Unschuldigen" war nur dank eines Verfassungsbruchs möglich. Das war der erste Vorbote der aufziehenden Veränderungen und ein Anzeichen des Wankelmutes mancher Richter.

Der nächste Schritt war, dass der Präsident drei legal gewählte Richter des Verfassungstribunals - sie waren noch vom Sejm der vorangegangenen Wahlperiode mit den Stimmen der $P O$ gewählt worden - nicht vereidigte. An ihre Stelle berief Duda drei mit den Stimmen der PiS neu gewählte Richter, unter anderem Mariusz Muszyński, einen ehemaligen Botschaftsangehörigen, der als persona non grata aus der Bundesrepublik Deutschland ausgewiesen worden war. Trotz Muszyńskis beruflicher Qualifikation als habilitierter Rechtswissenschaftler waren hier zwei Fakten von Bedeutung. Erstens erlaubte seine Vergangenheit nicht, ihn als untadeligen Charakter zu bezeichnen, was jedoch eine Anforderung an die Ausübung des Richteramtes ist, und zweitens wurde er auf einen Posten berufen, der bereits besetzt war. Das hinderte den Präsidenten allerdings nicht daran, ihn zu vereidigen. Drei solche »Richter-Double« gehören zurzeit dem Verfassungstribunal an. Die Folge ist die formelle Ungültigkeit der Urteile, wenn sich mindestens ein "Double« unter den urteilsprechenden Richtern befindet. Außer dem offenkundigen Glaubwürdigkeitsverlust, was das rechtsstaatliche Handeln des Verfassungstribunals betrifft, gibt es weitere Konsequenzen. Erstens kam es zu einem drastischen Rückgang der Rechtsfälle, die an das Verfassungstribunal gerichtet wurden; zudem werden sie immer langsamer bearbeitet. Zweitens kam es dazu, dass der Europäische Gerichtshof für Menschenrechte in Straßburg einem polnischen Staatsbürger Recht gab und ihm eine Entschädigung zuerkannte, weil dieser angezeigt hatte, dass seine Verfassungsklage von einer nicht rechtmäßig besetzten Richterschaft geprüft worden war, da ihr ein »Richter-Double« angehörte.

Zusammengefasst: Bereits in der ersten Phase der PiS-Regierung wuchs das rechtliche Chaos und wurden nicht nur fundamentale Grundsätze wie das Prinzip des demokratischen Rechtsstaates, das Prinzip des Handelns von staatlichen Organen auf der Grundlage und in den Grenzen des Rechtes, das Prinzip der Unabhän- gigkeit der Gerichte und der Richter und das Prinzip der Achtung der Rechte und der Freiheiten des Menschen gebrochen, sondern auch das grundlegende Prinzip des Vertrauens des Bürgers in die staatlichen Gewalten. Das Letztgenannte, das auf der Rechtsprechung des Verfassungstribunals in den 1990er Jahren gründet, schied Polen symbolisch und praktisch von der Willkür und Mittelmäßigkeit der kommunistischen Herrschaft in Polen bis 1989.

\section{Entscheidende Veränderungen}

Weitere Veränderungen betrafen die allgemeinen Gerichte. Die von der Regierung unter der Führung von Justizminister Zbigniew Ziobro lancierten Justizreformen wurden von Teilen der Öffentlichkeit und Politikern der Opposition schnell als »Deformen« bezeichnet. Zwei davon weisen besonders negative Aspekte auf: die Veränderungen beim Obersten Gericht und hier die Berufung einer Disziplinarkammer, quasi als Gericht über dem Gericht, und zweitens die Reform des Landesjustizrates (Krajowa Rada Sądownictwa - KRS).

Der Landesjustizrat wurde 1989 in das staatliche System der Republik Polen eingeführt. Dieser Typus von nicht Recht sprechenden Organen, die aber dem Schutz der Rechtsprechung dienen, begannen in Europa in der zweiten Hälfte des 20. Jahrhunderts zu entstehen. Aktuell treten sie in vielen europäischen Staaten auf, unter anderem in Italien, Frankreich, Portugal und der Slowakei. Die wesentliche Aufgabe des Landesjustizrates war es, über die Unabhängigkeit der Gerichte und der Richter zu wachen. Der Rat setzt sich aus Parlamentariern, dem Justizminister, dem Präsidenten des Obersten Verwaltungsgerichtes (Naczelny Sad Administracyjny), einer vom Staatspräsidenten bestimmten Person und schließlich 15 Richtern zusammen. Die PiS-Mehrheit im Sejm änderte das Gesetz über den Landesjustizrat. Die Amtszeit des Rates wurde verkürzt und neue Mitglieder berufen. Das war nicht verfassungskonform, vielmehr war es eine treue Kopie der Aktivitäten, die Ministerpräsident Viktor Orbán in Ungarn an den Tag legt. Nicht zuletzt hat PiS-Parteichef Jarosław Kaczyński den Polen wiederholt versprochen, dass »ein zweites Budapest an der Weichsel« entstehen werde. Ein weiterer politischer Schachzug à la Orbán, der von der PiS aufgenommen wurde, war, Richter in den vorgezogenen Ruhestand zu schicken. Wegen Diskriminierung (Frauen sollten mit 60 Jahren, Männer mit 65 Jahren pensioniert werden) wurde dies von der EU teilweise blockiert. Die wichtigste Änderung beim Landesjustizrat - und gleichzeitig die direkte Kollision mit der Verfassung - war, dass die Richter nicht wie bisher von einem Richtergremium berufen wurden, sondern vom Sejm. Dem polnischen staatlichen System und dem Prinzip der Gewaltenteilung entsprechend, sind die Legisla- 
tive, Exekutive und Judikative drei separate und unabhängige Gewalten. Ein kompliziertes Zusammenspiel von Einschränkungen und ausgleichenden Mechanismen gewährleistet den von Montesquieu gesetzten Standard. Die gegen die Verfassung verstoßende politische Wahl der Richter in den Landesjustizrat zerstörte den Standard. Zunächst fanden sich keine Willigen und es schien, als würden die Neuwahlen zum Landesjustizrat von der Richterschaft boykottiert. Allerdings meldeten sich im letzten Moment einige Waghalsige, die bereit waren, die Verfassung zu brechen. Ein Teil von ihnen war mit Justizminister Ziobro verbandelt, ein Teil wäre in normaler Situation nie in den Landesjustizrat berufen worden, beispielsweise wegen Disziplinarproblemen. Es gab keine Kandidaten aus den Berufungs-, Verwaltungs- und Militärgerichten sowie dem Obersten Gericht. Politisch opportune Richter der Gerichte der unteren Instanzen wurden allerdings berufen und sandten somit den anderen Richtern eine deutliche Botschaft: Es lohnt sich, den aktuellen Machthabern zu gefallen, auch wenn das mit einem Verfassungsbruch bezahlt werden muss. Von diesem Moment an ist in Polen das Rechtssystem durch einen spezifischen Dualismus gekennzeichnet. In dem einen Bereich sind die Verfassung der Republik Polen und das verfassungsgemäße Recht angesiedelt, das von der Mehrheit der Richter in Polen angewendet wird. Dem Grundsatz der Hierarchie der Rechtsnormen gemäß verweigern diese Richter, Gesetze anzuwenden, die mit der polnischen Verfassung und internationalen Verträgen nicht vereinbar sind. Den anderen Bereich des Rechtssystems bestimmen die Richter, die das neue Recht anwenden und die Vorschriften der Verfassung außer Acht lassen. Diese Gruppe erwarten Beförderungen, hohe finanzielle Zulagen und zusätzliche Posten. Die Gruppe der rechtschaffenen Richter wehrt sich dagegen, an der Ausübung ihrer Tätigkeit gehindert zu werden, sowie gegen Disziplinarvorwürfe und Hassrede in den sozialen Medien. Das Privatleben eines Teils von ihnen wird in den regierungsnahen öffentlichen Medien kommentiert. Unlängst wurde die Existenz und Tätigkeit des neuen Landesjustizrates von einem internationalen Expertengremium klar kommentiert: Er wurde nach vorangegangener Aussetzung aus dem European Networks of Councils for the Judiciary $(E N C J)$ ausgeschlossen.

\section{Die Disziplinarkammer des Obersten Gerichtes}

Außer dem Signal, dass die Machthaber diejenigen Richter belohnen, die zum Verfassungsbruch bereit sind, gab es noch eine weitere Botschaft: Richter, die die Verfassung achten, haben Sanktionen und Schikanen zu erwarten - ebenso wie Richter, die gegen die eingeführten Änderungen protestieren. Einer der Richter des aktuellen Verfassungstribunals, Stanisław Piotrowicz, ehemaliger Staatsanwalt der Volksrepublik Polen und Sejmabgeordneter für die PiS (2015 bis 2019), hat die Erwartungen der Legislative und Exekutive an die polnischen Richter präzise formuliert. Im Zusammenhang mit einem Entwurf des Gesetzes über den Landesjustizrat sagte er 2017 vor dem Sejm, die Reform diene nicht nur strukturellen, sondern auch personellen Veränderungen, denn es solle zu einer »qualitativen Veränderung bei den Richtern« kommen, die sich durch eine »dienstbare Mentalität« legitimieren sollen. Für die Garantie der "dienstbaren Mentalität der Richter«, die die verfassungsmäßige Erfordernis der Unabhängigkeit ersetzen sollte, sollte die neu berufene Disziplinarkammer des Obersten Gerichtes sorgen.

Die Konstruktion der Disziplinarkammer, die theoretisch in das Oberste Gericht eingegliedert ist, praktisch aber ein übergeordnetes Gericht darstellt, ist für die polnische Judikative ohne Beispiel. Die Richter der Kammer erhalten ein im Vergleich zu den anderen Richtern des Obersten Gerichtes 40 Prozent höheres Gehalt. Sie unterstehen nicht der Aufsicht des Ersten Präsidenten des Obersten Gerichtes und werden alle vom neuen Landesjustizrat berufen. Ihnen werden Disziplinarangelegenheiten angetragen, die nicht nur Richter, sondern auch Staatsanwälte, Rechtsanwälte oder Rechtsberater betreffen. Rasch ergriff die Disziplinarkammer Maßnahmen gegen widerständige Richter und Staatsanwälte, die sich dem Verfassungsbruch widersetzten. Die Tätigkeit der Disziplinarkammer allein brachte allerdings nicht die erwünschten Folgen. Die Regierung entschied sich daher für einen weiteren Schritt.

\section{Das "Maulkorbgesetz»}

Anfang 2020 trat ein neues Gesetz in Kraft, das ein für alle Mal den Richtern die Unabhängigkeit nehmen und sie zum Schweigen bringen sollte. Die Absichten des Gesetzgebers waren so offensichtlich, dass es umgehend als »Maulkorbgesetz« bezeichnet wurde. Auf seiner Grundlage wurden neue Vorschriften eingeführt, welche die Durchführung eines Disziplinarverfahrens gegenüber Richtern anordnen, die

- die Arbeit der Justizorgane erschweren oder unmöglich machen,

- die Wirksamkeit der Ernennung von Richtern oder die Vollmacht der Organe der Republik Polen infrage stellen,

- in einer Weise öffentlich tätig sind, die sich nicht mit dem Prinzip der Unabhängigkeit der Gerichte und der Richter vereinbaren lässt.

Offenkundig zielte das Gesetz auf Richter, die sich der Zerstörung der Judikative und des Rechtsstaates widersetzten. Einerseits geboten die neuen Vorschriften (unter Androhung von Disziplinarmaßnahmen, die den Aus- 
schluss aus dem Richterstand zur Folge haben können), die unter Verfassungsbruch berufenen Richter als rechtsstaatlich legitimiert anzuerkennen und die nicht verfassungsgemäß besetzten Organe als rechtmäßig. Andererseits wurden die Vorschriften so weit gefasst und so uneindeutig formuliert, dass es offenkundig war, dass sie zur Einschüchterung eines jeden Richters unter beliebigem Vorwand dienen können. Das Berufsleben jedes polnischen Richters ist mit einem beständigen Konflikt verbunden: Entweder wird er in einem nicht korrekt besetzten Richtergremium Urteile sprechen - womit er einen Verfassungsbruch begeht - oder er lehnt es in dieser Situation ab, Recht zu sprechen, was zur Folge hat, dass ein Disziplinarverfahren gegen ihn eingeleitet wird, dies wiederum mit der Folge, ihn von der Rechtsprechung abzuziehen und ihn in nicht ferner Zukunft womöglich aus dem Richterstand auszuschließen. Dem polnischen Richter wird hiermit gestattet, zwischen Pest und Cholera zu wählen.

\section{Der zivilgesellschaftliche Aspekt}

Am 7. Mai 2021 sprach der Europäische Gerichtshof für Menschenrechte (EGMR) ein Schlüsselurteil in einer Angelegenheit des polnischen Unternehmens Xero Flor, das Rollrasen herstellt. Das Unternehmen hatte vom Staat eine Entschädigung für die Zerstörung seines Anbaus durch Wildschweine gefordert. Allerdings wurden die 100 Prozent Entschädigung auf 75 Prozent mittels einer Verfügung (also eines Rechtsaktes unterhalb der Gesetzesebene) reduziert. Nach verlorenem Prozess und Ausschöpfung des Gerichtsweges zog Xero Flor vor das Verfassungstribunal. Da dieses keine Verletzung der Bürgerrechte sah, ging die Angelegenheit weiter an die letztmögliche Instanz, den Europäischen Gerichtshof für Menschenrechte in Straßburg. Dieser gab dem Unternehmen nicht nur Recht, sondern befand auch, dass es zu einer zweifachen Rechtsverletzung gekommen war. Erstens hätte Xero Flor 100 Prozent Entschädigung erhalten müssen. Zweitens wurde das Recht auf einen fairen Gerichtsprozess verletzt, da sich unter den verhandelnden Richtern des Verfassungstribunals ein »Richter-Double« befand, das Richtergremium also unkorrekt besetzt war. Die Antwort des polnischen Justizministers ließ nicht lange auf sich warten: Er wandte sich an das Verfassungstribunal, um die Vereinbarkeit von Art. 6 der Europäischen Menschenrechtskonvention mit der polnischen Verfassung prüfen zu lassen. Ein tragisch-komischer Aspekt bei dieser ganzen Angelegenheit ist nicht nur die Übereinstimmung des Art. 6 mit Art. 45 der polnischen Verfassung (das Recht auf ein rasches, faires und transparentes Verfahren durch ein unabhängiges und unparteiisches Gericht), sondern auch, dass das Muster für die Verfassung hier Art. 6 der Europäischen Menschenrechtskonvention war.

\section{Ähnlichkeiten und Unterschiede}

Eine beliebte rhetorische Phrase der PiS-Politiker und von Justizminister Ziobro ist bei der Verteidigung der Pseudo-Reformen die Versicherung, dass sie im Wesentlichen dem System entsprechen, das in den westeuropäischen Staaten gilt. Hier werden meistens Frankreich, Deutschland, Spanien und Portugal angeführt. Das Land, das in dieser Reihe am häufigsten genannt wird, ist die Bundesrepublik Deutschland.

Das System der Richterberufung in Polen und in der Bundesrepublik Deutschland zu vergleichen, ist kompliziert und eine eher halsbrecherische Angelegenheit. Wesentliche Unterschiede treten schon auf der Ebene der akademischen Ausbildung auf. In Polen endet die universitäre Ausbildung mit dem Magisterexamen und nicht mit zwei Staatsexamen wie in Deutschland, die vollständige Unabhängigkeit garantieren. Bevor verglichen wird, muss auch berücksichtigt werden, dass Polen ein zentralistischer Staat ist und nicht ein föderativer wie Deutschland. Generell lässt sich sagen, dass das deutsche System der Richterberufung aus vielen Schritten besteht, u. a. Begutachtungen durch verschiedene Gremien (zum Beispiel Repräsentationen der Bundesländer), für die es keine Entsprechung im polnischen Rechtssystem gibt. In Polen sieht der Weg kürzer aus. Bewerbungen für das Richteramt, also von Personen, die das Referendariat absolviert und das Examen abgelegt haben, werden vom Landesjustizrat geprüft und begutachtet. Danach werden die Kandidaten dem Präsidenten der Republik Polen vorgestellt, der die Richter auf unbefristete Zeit (auf Lebenszeit) beruft. Ein wesentlicher, von der PiSRegierung eingeführter Aspekt ist die politische Instrumentalisierung der Richter, die in den Landesjustizrat gehen. Diese sind sich dessen bewusst, dass ihre Zugehörigkeit zu diesem wichtigen Organ nur aufgrund des Verfassungsbruchs möglich ist, und sie passen auf, dass eigenständige und verfassungstreue Richter nicht aufsteigen. Diesen erlaubt ihre Verfassungstreue nicht, den Landesjustizrat zu akzeptieren, dem Richter angehören, welche die Verfassung brechen. Das zieht weitere Implikationen nach sich, beispielsweise wird infrage gestellt, dass die Berufung der auf diese Weise bestimmten Richter durch den Präsidenten den Berufungsprozess legalisiert. Die These, dass die präsidiale Berufung die Illegalität der Tätigkeiten des Landesjustizrates aufhebt, ist nicht nur eine stark lancierte politische These, sondern nach Auffassung der PiS auch ein Dogma, das nicht zur Diskussion steht. Die Infragestellung dieser These sei ein Angriff auf das präsidiale Vorrecht und die polnische Souveränität. Verfolgt man die These weiter, es sei legal und rechtsstaatlich, dass der Präsident Personen ins Richteramt beruft, die die verfassungsmäßigen Anforderungen nicht erfüllen, müsste man konsequenterweise anerkennen, dass dem Präsidenten irgendwelche 
Super-Befugnisse zustehen, die in der Verfassung nicht aufgeführt werden. Der Präsident selbst wäre dann ein Organ, das nicht in der Konstruktion des demokratischen Staates mit Kabinettsystem verankert wäre. Der Präsident der Republik Polen ist jedoch ein Element der ausführenden Gewalt, die im Rahmen des Prinzips der Dreiteilung der Gewalten realisiert wird. Ganz sicher ist er kein übergeordnetes Organ, das mit "Supermacht « ausgestattet ist, die in der Verfassung unbekannt ist und das Konzept der Gesetzlichkeit der Tätigkeiten der Machtorgane und das Prinzip des demokratischen Rechtsstaates zerstört.

Die deutliche Mehrheit der polnischen Richter bleibt der Verfassung treu und lehnt es daher ab, mit den vom neuen Landesjustizrat befürworteten Richtern gemeinsam eine Verhandlung zu führen. Damit scheiterten die Erwartungen der PiS, dass alle die nicht verfassungsgemäßen, "politisch" berufenen Richter vergessen und diese bei schweigender Akzeptanz der anderen Richter still und heimlich in der Judikative aufgehen. Der Konflikt bleibt bestehen.

\section{Neueste Ankündigungen}

Wie bereits erwähnt, wurde die Entscheidung der Richterin Rosario Silva de Lapuerta am Europäischen Gerichtshof vom Juli 2021, dass die Tätigkeit der Disziplinarkammer des Obersten Gerichtes einzustellen sei, bis heute nicht in Polen umgesetzt. Zwar kündigte die Erste Präsidentin des Obersten Gerichtes, Małgorzata Manowska, nach zunächst hochmütigen Aussagen, dass die Disziplinarkammer weiterarbeiten werde, deren Einstellung an, aber es passierte nichts dergleichen. Die Polen auferlegte Strafe wird von der Regierung auf widersprüchliche Weise kommentiert. Zwar werden Ankündigungen gemacht, die Kammer aufzulösen, aber es werden auch Appelle wiederholt, sich damit nicht zu beeilen bzw. das Bestehen der Kammer zu verteidigen. Auf das Urteil des EuGH, dass die Disziplinarkammer kein Gericht im Sinne des EU-Rechtes sei, reagierte das Verfassungstribunal, indem es die Grundlagen der EUVerträge infrage stellte. Die Verschärfung des Konfliktes mit der EU nach dem unglücklichen Urteil des Verfassungstribunals veranlasste den PiS-Chef und Vizeministerpräsidenten Jarosław Kaczyński zu der Ankündigung, die Disziplinarkammer vollständig aufzulösen. Das bedeutet aber nicht unbedingt eine Verbesserung der Situation. In einem aktuellen Interview mit dem Sender »Radio RMF 24.pl« kündigte Kaczyński revolutionäre Veränderungen in der Justiz an. Unter anderem sollen Gerichte der untersten Stufe und die Berufungsgerichte (oberste Stufe, ohne Oberstes Gericht) aufgelöst und durch Bezirksgerichte und vollkommen neue Regionalgerichte ersetzt werden. Die Regionalgerichte sollen den Großteil der Kompetenzen des Obersten Gerichtes übernehmen, dessen Anzahl an Richtern deutlich (auf knapp 20) reduziert werden soll. Dem Obersten Gericht bliebe die rätselhafte Aufgabe, die »Rechtsprechung zu ordnen«. Die Grundlagen dieser Reformen sind allerdings nicht mit dem Grundgesetz vereinbar. Sie werden also nicht dazu dienen, den Konflikt zu beenden und die Angelegenheiten tatsächlich zu ordnen.

Unabhängige Beobachter stimmen in ihren neuesten Diagnosen überein: Nach fünf Jahren Justizreform hat sich das Chaos in den Gerichten nur verschlimmert. Die Gerichtsverfahren verlängern sich zunehmend und die Verhandlungsführung durch Richter mit unklarem Status ist eine Grundrechtsverletzung, und zwar des Rechtes auf einen fairen Gerichtsprozess. Die angekündigten Änderungen werden nicht dazu dienen, die Arbeit der Gerichte effektiver zu machen. Ihr Ziel ist es, rechtschaffene Richter abzuziehen, insbesondere die in der ersten Reihe, und die übrigen einzuschüchtern.

\section{Fazit}

Wenn regierungskritische Politiker, Wissenschaftler und Beobachter des gesellschaftlichen Lebens in Polen über den Beginn des Konfliktes der Judikative mit der Exekutive und der Legislative sprechen, nennen sie das Jahr 2015 und die Entscheidung des Präsidenten Andrzej Duda, drei legal gewählte Richter nicht zu vereidigen und an ihre Stelle drei illegal gewählte »Richter-Double« zu setzen. Jedoch sollte die Entstehung des Konfliktes früher datiert werden, denn in die Wahlen von 2015 ging die PiS mit dem Entwurf für eine neue Verfassung. Obwohl also die Verfassung von 1997 nur die Möglichkeit vorsieht, Veränderungen am geltenden Grundgesetz vorzunehmen, wagte es die $P i S$, einen völlig neuen Entwurf zu erstellen.

In diesem gab es keine Dreiteilung der Gewalten und die gesamte Macht sollte beim Präsidenten liegen, der weder einer politischen Verantwortung unterlag noch einer konstitutionell-rechtlichen. Über den polnischen Präsidenten, der bei sich den größten Teil der Macht konzentrieren sollte, sollten nur Gott und die Geschichte richten. Diese archaische Konstruktion passt eher ins 19. Jahrhundert als in das gegenwärtige Polen, jedoch stellen sich so Kaczyńskis Träume vom politischen System in Polen dar. Meine Hoffnung ist, dass sie sich nie erfüllen werden, denn sie sind die Vision eines autoritären Staates, weit entfernt vom Konzept des demokratischen Rechtsstaates, der die Rechte des Menschen und aller Minderheiten achtet, wie es in der polnischen Verfassung steht. Wie sehen also die Zukunftsprognosen aus? Sie sind leider nicht sonderlich optimistisch. Der Konflikt wird weitergehen. Wir wissen bereits, dass die PiS sogar um den Preis des EU-Austritts bereit wäre, ihre Macht zu sichern. Die Reaktionen des polnischen Ministerpräsidenten und des Justizministers (bzw. der 
gesamten Regierungsmannschaft) auf die Entscheidung des EuGH, Polen im Zusammenhang mit der Disziplinarkammer mit Zwangsgeld zu belegen, müssen als inadäquat für dieses Problem bewertet werden. Die Aussagen von Ministerpräsident Mateusz Morawiecki in einem kürzlich veröffentlichten Interview in der "Financial Times«, die Verknüpfung der Rechtsstaatlichkeit mit der Auszahlung von EU-Geldern und deren gegenwärtiger Blockade setze »Polen die Pistole auf die Brust" und sei »der dritte Weltkrieg«, wurden in Polen ungläubig aufgenommen. Der hysterische Ton der Äußerungen Morawieckis ist der unglückliche Versuch, die Schwäche des Regierungshandelns zu maskieren und Ausdruck mangelnder Argumente im Konflikt mit der Europäischen Union. Sicherlich sprechen dieser Art Äußerun- gen einen Teil der polnischen Wähler an, der Rest der Gesellschaft nimmt sie jedoch distanziert auf. Die PiS verliert allmählich an Unterstützung, und ich hoffe, dass sich daraus ein dauerhafter Trend entwickelt, denn jeder weitere Tag entblößt die Beschränktheit der Argumentation des Ministerpräsidenten und seine leere Emotionalität. Daher fasst die PiS die Appelle der Europäischen Union, den polnischen Gerichten Unabhängigkeit zu garantieren, auch als Angriff auf die polnische Souveränität auf und übertönt sie mit der abstrusen Rede, dass das polnische Gerichtswesen sich nicht von dem westlicher Staaten unterscheide und dass es vielmehr darum gehe, dass die EU die Polen nicht mag.

Übersetzung aus dem Polnischen: Silke Plate

Über die Autorin

Dr. Bogna Baczyńska ist wissenschaftliche Mitarbeiterin an der Fakultät für Recht und Verwaltung der Universität Stettin (Wydział Prawa i Administracji, Uniwersytet Szczeciński) und Kommentatorin u. a. für tvn24.pl. Zu ihren Forschungsgebieten gehören Verfassungsrecht (national und vergleichend), EU-Recht, Menschen- und Bürgerrechte.

DOKUMENTATION

\section{Plenardebatte im Europäischen Parlament zur Rechtsstaatlichkeit in Polen, 19. Oktober 2021 (Auszüge)}

\section{Plenardebatte im Parlament der Europäischen Union: Die Krise im Zusammenhang mit der Rechtsstaatlichkeit in Polen und der Vorrang des Unionsrechts (Aussprache)}

\section{Ursula von der Leyen, Präsidentin der Europäischen Kommission:}

Mr President, almost 40 years ago, in December 1981, the communist regime in Poland imposed martial law. Many members of Solidarność, the independent trade union, and of other groups were put in jail, simply because they stood up for their rights.

The people of Poland wanted democracy, like millions of other Europeans from Budapest to Tallinn to East Berlin. They wanted the freedom to choose their government, they wanted free speech and free media, they wanted an end to corruption, and they wanted independent courts to protect their rights. The people of Central and Eastern Europe wanted to join the European family of free people, a strong community of values and democracy. Because that is what Europe is about and that is what Europe stands for.

The recent ruling of the Polish constitutional court puts much of this into question. We have been concerned about the independence of the judiciary for some time. Judges have seen their immunity being lifted and have been driven out of office without justification, and this threatens judicial independence, which is a basic pillar of the rule of law.

We have taken a number of measures. We continue to have a regular dialogue. But unfortunately the situation has worsened. And this is not only the Commission's opinion, this is what has been confirmed by the European Court of Justice and the European Court of Human Rights. And now this has culminated in the most recent ruling of the Polish constitutional court.

The European Commission is, at the moment, carefully assessing this judgment, but I can already tell you today that I am deeply concerned. This ruling calls into question the foundations of the European Union. It is a direct challenge to the unity of the European legal order. Only a common legal order provides equal rights, legal certainty, mutual 
trust between Member States and, therefore, common policies. This is the first time ever that the court of a Member State finds that the EU treaties are incompatible with the national constitution.

This has serious consequences for the Polish people because the ruling has a direct impact on the protection of the judiciary. The ruling undermines the protection of the judicial independence, as guaranteed by Article 19 of the Treaty and as interpreted by the European Court of Justice. Without independent courts, people have less protection and consequently their rights are at stake.

Polish people must be able to rely on fair and equal treatment in the judicial system, just like any other European citizen. In our Union, we all enjoy the same rights and this basic principle fundamentally impacts people's lives because, if European law is applied differently in Grenoble or Göttingen or Gdańsk, EU citizens would not be able to rely on the same rights everywhere.

When joining the European Union, the Polish people put their trust in the European Union. They expected the European Union to defend their rights, and rightly so. The Commission is the guardian of the Treaty. It is my Commission's duty to protect the rights of EU citizens wherever they live in our Union. The rule of law is the glue that binds our Union together. It is the foundation of our unity. It is essential for the protection of the values on which our Union is founded: democracy, freedom, equality and respect for human rights. This is what all 27 Member States have signed up to as part of this Union, as sovereign countries and free people.

We cannot - and will not - allow our common values to be put at risk. The Commission will act, and the options are all known. The first option are infringements, where we legally challenge the judgment of the Polish constitutional court.

Another option is the conditionality mechanism and other financial tools. The Polish Government has to explain to us how it intends to protect European money, given this ruling of their constitutional court because, in the coming years, we will be investing EUR 2100 billion with the multiannual budget and the NextGenerationEU recovery programme. This is European taxpayers' money and, if our Union is investing more than ever to advance our collective recovery, we must protect the Union's budget against breaches of the rule of law.

The third option is the Article 7 procedure. This is the powerful tool in the Treaty, and we must come back to it because, let me remind you, the Polish constitutional court that today has cast doubts on the validity of our Treaty is the same court that, under Article 7, we consider not to be independent and legitimate. This, in many ways, comes full circle.

I deeply regret that we find ourselves in this situation. I have always been a proponent of dialogue and I will always be. This is a situation that can and must be resolved. We want a strong Poland in a united Europe. We want Poland to be at the heart of our debates in building a common future. Poland has a stake in Europe. Together, we can build a Europe that is strong and confident in a world where other big powers become more and more assertive.

Europe has benefited from Poland's unique experience so much. Without the people of Poland, our European journey would have been very different. When Karol Wojtyła, as Pope John Paul II, went to his homeland, he changed European history forever. When Lech Wałęsa, with a scattered group of trade unionists, overcame a mighty army, we saw the beginning of the fall of the Iron Curtain. And when President Lech Kaczyński ratified the Lisbon Treaty, together with the Charter of Fundamental Rights, he reaffirmed Poland's commitment to our values. Polish people have played a fundamental role in making our Union whole, in enabling their homeland to thrive as a vital part of our Union, and they will always be.

Polsko, jesteś i zawsze będziesz w sercu Europy! Niech żyje Polska, niech żyje Europa!

Poland, you are, and you will always be, at the heart of Europe. Long live Poland and long live Europe! [im Original Englisch, Anm.d.Red.]

\section{Mateusz Morawiecki, Ministerpräsident der Republik Polen:}

Herr Präsident! Frau Präsidentin! Sehr geehrte Abgeordnete! Ich stehe hier heute vor Ihnen im Parlament, um unseren Standpunkt in einigen grundsätzlichen Fragen darzulegen, die ich für grundlegend für die Zukunft der Europäischen Union halte. Und nicht nur für die Zukunft Polens, sondern gerade für die Zukunft unserer ganzen Union. $[\ldots]$

Politik muss sich auf Grundsätze stützen. Der wichtigste Grundsatz, den wir in Polen anerkennen und der die Grundlage der Europäischen Union ist, ist das Prinzip der Demokratie. Daher können wir nicht schweigen, wenn unser Land u. a. in diesem Saal ungerecht und parteiisch angegriffen wird. Die Spielregeln müssen für alle gleich sein und ihre Befolgung ist die Pflicht aller, also auch der Institutionen, die in den Verträgen beschlossen wurden. Darauf beruht die Rechtsstaatlichkeit. Unzulässig ist es, die Kompetenzen zu erweitern und mit der Methode der vollendeten Tatsachen zu handeln. Unzulässig ist es, anderen eine Entscheidung ohne rechtliche Grundlage aufzuzwingen. So ist es noch unzulässiger, für dieses Ziel die Sprache der finanziellen Erpressung einzusetzen, von Strafen zu sprechen oder 
noch weiter gehende Worte gegenüber manchen Mitgliedsstaaten zu verwenden. Ich weise die Sprache der Bedrohung, der Drohungen und Erpressungen zurück. Ich akzeptiere nicht, dass Politiker Polen erpressen und erschrecken, dass Erpressung eine Methode gegenüber einem der Mitgliedsländer geworden ist. So sieht tritt Demokratie nicht aus. [...]

Hohes Haus, nun einige Worte zur Rechtsstaatlichkeit. Über die Rechtsstaatlichkeit kann man ja viel sagen, und jeder wird den Begriff etwas anders verstehen. Aber ich denke, dass die Mehrheit von uns darin übereinstimmt, dass von Rechtsstaatlichkeit nicht die Rede sein kann ohne einige Bedingungen, ohne das Prinzip der Gewaltenteilung, ohne Unabhängigkeit der Gerichte, ohne Beachtung des Grundsatzes, dass jede Gewalt Beschränkungen hat, beschränkte Kompetenzen, und auch ohne Wahrung der Hierarchie der Rechtsquellen. Das EU-Recht gilt vor nationalem Recht bis auf Gesetzesebene in den Bereichen, die der Kompetenz der Europäischen Union übertragen wurden. Dieses Prinzip gilt in allen Mitgliedsländern, aber das höchste Recht bleibt die Verfassung.

Wenn die Institutionen, die in den Verträgen bestimmt werden, ihre Kompetenzen überschreiten, müssen die Mitgliedsstaaten Instrumente haben, um zu reagieren. Die Europäische Union ist eine große Errungenschaft der Staaten Europas und ein starkes wirtschaftliches, politisches und gesellschaftliches Bündnis. Sie ist die stärkste, am weitesten entwickelte internationale Organisation in der Geschichte. Aber die Europäische Union ist kein Staat. Staaten sind die 20 [sic!] Mitgliedsstaaten der Union. Es sind die Staaten, die die europäischen Souveräne bleiben, sie sind die Herren der Verträge, und Staaten bestimmen den Kompetenzbereich, der der Europäischen Union übertragen, zuerkannt wird. In den Verträgen haben wir der Union einen sehr großen Kompetenzbereich übertragen, aber wir haben ihr nicht alles übertragen. Viele Bereiche des Rechtes bleiben in der Kompetenz der Nationalstaaten. Wir bezweifeln nicht den Vorrang des EU-Rechtes vor den nationalen Gesetzen in allen Bereichen, in denen die Kompetenzen von den Mitgliedsstaaten der Union übertragen wurden. Aber ähnlich wie die Verfassungsgerichte in vielen anderen Ländern stellt das polnische Verfassungstribunal die Frage, ob das Monopol des Gerichtshofes der Europäischen Union, die tatsächlichen Grenzen dieser Kompetenzübertragung zu bestimmen, die richtige Lösung ist.

[...]

Ihr Lieben, wenn ihr aus Europa einen nationslosen Superstaat machen wollt, dann holt erst die Zustimmung aller Staaten und Gesellschaften Europas ein. Ich wiederhole es noch einmal: Das höchste Recht der Republik Polen ist die Verfassung. Sie gilt vor allen anderen Rechtsquellen. Von diesem Prinzip kann kein polnisches Gericht zurücktreten, kein polnisches Parlament und keine polnische Regierung. Es soll allerdings unterstrichen werden, dass das polnische Verfassungsgericht nie festgestellt hat, auch nicht in seinem letzten Urteil, dass die Vorschriften des Vertrags über die Europäische Union in Gänze unvereinbar mit der polnischen Verfassung sind. Im Gegenteil, Polen achtet die Verträge. Daher hat ja auch das polnische Verfassungstribunal festgestellt, dass unvereinbar mit der Verfassung eine einzige, sehr konkrete Interpretation einiger Vorschriften des EU-Vertrages ist, die aus dem letzten Urteil des Gerichtshofes der Europäischen Union erfolgt.

Um das zu erklären komme ich jetzt zum nächsten Teil meiner Ausführungen, zu den Gefahren für das ganze Gesellschaftssystem, wenn der Status eines Richters durch einen anderen Richter infrage gestellt wird. Laut Interpretation des Gerichtshofes in Luxemburg sollen die Richter der polnischen Gerichte verpflichtet sein, den Grundsatz des Vorrangs des EU-Rechtes vor den nationalen Vorschriften im Rang eines Gesetzes anzuwenden, was keinen Zweifel weckt, aber auch die Verfassung und die Urteile des eigenen Verfassungstribunals zu brechen. Diese Interpretation zu akzeptieren, kann in der Folge dazu führen, zu akzeptieren, dass Millionen Urteile, die in den letzten Jahren von polnischen Gerichten gesprochen wurden, willkürlich infrage gestellt und Tausende Richter ihres Amtes enthoben werden können. Millionen Urteile! Das würde gegen den Grundsatz der Unabhängigkeit, Unkündbarkeit sowie der Stabilität und des garantierten Rechtes auf ein Gerichtsverfahren gehen, der sich direkt aus der polnischen Verfassung ergibt. Ist Ihnen nicht bewusst, wozu diese Entscheidung führen kann? Will wirklich einer von Ihnen in Polen Anarchie, Chaos und Rechtlosigkeit einführen?

[...]

Der Erfolg der europäischen Integration beruhte darauf, dass das Recht aus den Mechanismen abgeleitet wurde, die unsere Staaten in anderen Bereichen verbinden. Der Versuch, dieses Modell um 180 Grad zu wenden, Integration mit Hilfe von Rechtsmechanismen aufzuzwingen, ist eine Abkehr von den Prinzipien, die der Ursprung des Erfolgs der Europäischen Gemeinschaft waren.

[...]

Die uns erreichenden paternalistischen Belehrungen über Demokratie und Rechtsstaatlichkeit, darüber, wie wir unser Vaterland zu gestalten haben, dass wir falsche Entscheidungen treffen, dass wir zu unreif sind, dass unsere Demokratie angeblich jung ist, ist eine fatale Richtung des Narrativs, das von manchen vorgeschlagen wird.

$[\ldots]$ 
Polen achtet die Prinzipien der Europäischen Union. Es lässt sich nicht einschüchtern und erwartet einen Dialog.

Um den Prozess dieses Dialogs zu optimieren, lohnt es sich, institutionelle Veränderungen vorzuschlagen. Die Zukunft Europas kann unser gemeinsamer Erfolg sein. Für einen dauerhaften Dialog, der den Grundsatz der checks and balances erfüllt, kann eine Kammer des Europäischen Gerichtshofes eingerichtet werden, der Richter angehören, die von den Verfassungsgerichten der Mitgliedsländer bestimmt werden. Ich mache Ihnen heute diesen Vorschlag. Die letzte Entscheidung muss beim demos und bei den Staaten liegen, aber die Gerichte sollten eine solche Plattform haben, um einen gemeinsamen Nenner zu suchen. [im Original Polnisch, Anm.d.Red.]

$[\ldots]$

\section{Ursula von der Leyen, Präsidentin der Europäischen Kommission:}

[...] Diese Debatte zeigt, wie ernst die Situation ist. Es ist, wenn wir auf den Kern der Debatte gehen und nicht versuchen, durch andere Debatten abzulenken, ein einmaliger Vorgang, dass ein Verfassungsgericht eines Mitgliedstaates Artikel des europäischen Vertrages infrage stellt. Das trifft mitten ins Mark der Rechtsstaatlichkeit, das hat es so noch nie gegeben, und darüber diskutieren wir. Hinzu kommt, dass dieses Verfassungsgericht, das die Axt an die europäischen Verträge legt, selbst in seiner Legitimität infrage steht. Das zeigt die ganze Tragweite! Darum geht es hier heute bei dem, worüber wir diskutieren.

(Zwischenrufe)

Ich habe die Handlungsempfehlungen und Möglichkeiten bei diesem Gerichtsurteil, das ja nur wenige Tage alt ist, die der Kommission und damit auch den Mitgliedstaaten und dem Parlament zur Verfügung stehen, dargelegt. Es ist hier - und darauf möchte ich auch eingehen - mehrfach die Frage nach dem Aufbaufonds gestellt worden. Auch hier sind die Regeln ganz klar. Wir haben festgelegt, und zwar gesetzlich: Es gibt Investitionen, aber die sind gekoppelt an Reformen. Die Reformen müssen die länderspezifischen Empfehlungen erfüllen. Und eine lang währende länderspezifische Empfehlung für Polen ist die Wiederherstellung der Unabhängigkeit der Justiz. Dazu zählen der Abbau der Disziplinarkammer, der Abbau des Disziplinarregimes, die Wiedereinsetzung der unrechtmäßig entlassenen Richterinnen und Richter. Das ist die Grundvoraussetzung. Ich habe eben gehört, dass Sie das planen. Tun Sie es!

Hohes Haus! Wir werden die Rechtsstaatlichkeit und die Verträge der Europäischen Union verteidigen - mit allen Mitteln. Diese Rechtsstaatlichkeit sieht aber klare Prozessschritte vor, und wir werden sie einhalten: Informationsgewinnung, Rede und Gegenrede, Stellungnahme und Gegenstellungnahme - all das verlangt der Europäische Gerichtshof. Dann haben wir einen Fall richtig aufgebaut, und dann sind wir auch erfolgreich, wie es gerade die jüngsten Urteile des Vertragsverletzungsverfahrens gezeigt haben. Wir haben noch nie ein Verfahren zur Rechtsstaatlichkeit verloren, und dabei wird es bleiben. Deshalb die Gründlichkeit, und ich bin sicher, dass dieses Hohe Haus mich dabei unterstützen wird.

Ja, die Demokratie und die Rechtsstaatlichkeit sind langsamer als die Autokratien, weil sie alle anhören, bevor ein Urteil gefällt wird oder eine Lösung gefunden wird. Aber genau das unterscheidet uns ja von den Autokraten und den Diktatoren dieser Welt. Die Verfahren sind fair und begründet und legitim und kraftvoll. Deshalb ist die europäische Rechtsordnung auch so überzeugend, und wir werden sie verteidigen - mit den Menschen in Polen. [im Original Deutsch, Anm.d.Red.]

Übersetzung aus dem Polnischen: Silke Plate

Quelle: Europäisches Parlament. https://www.europarl.europa.eu/doceo/document/CRE-9-2021-10-19-ITM-002_ DE.html (abgerufen am 28.10.2021). 


\section{Bewertungen der Tätigkeit der Justiz durch die polnische Bevölkerung}

Grafik 1: Bewertungen der Tätigkeit des Verfassungstribunals (Dezember 2002 - September 2021, \%)

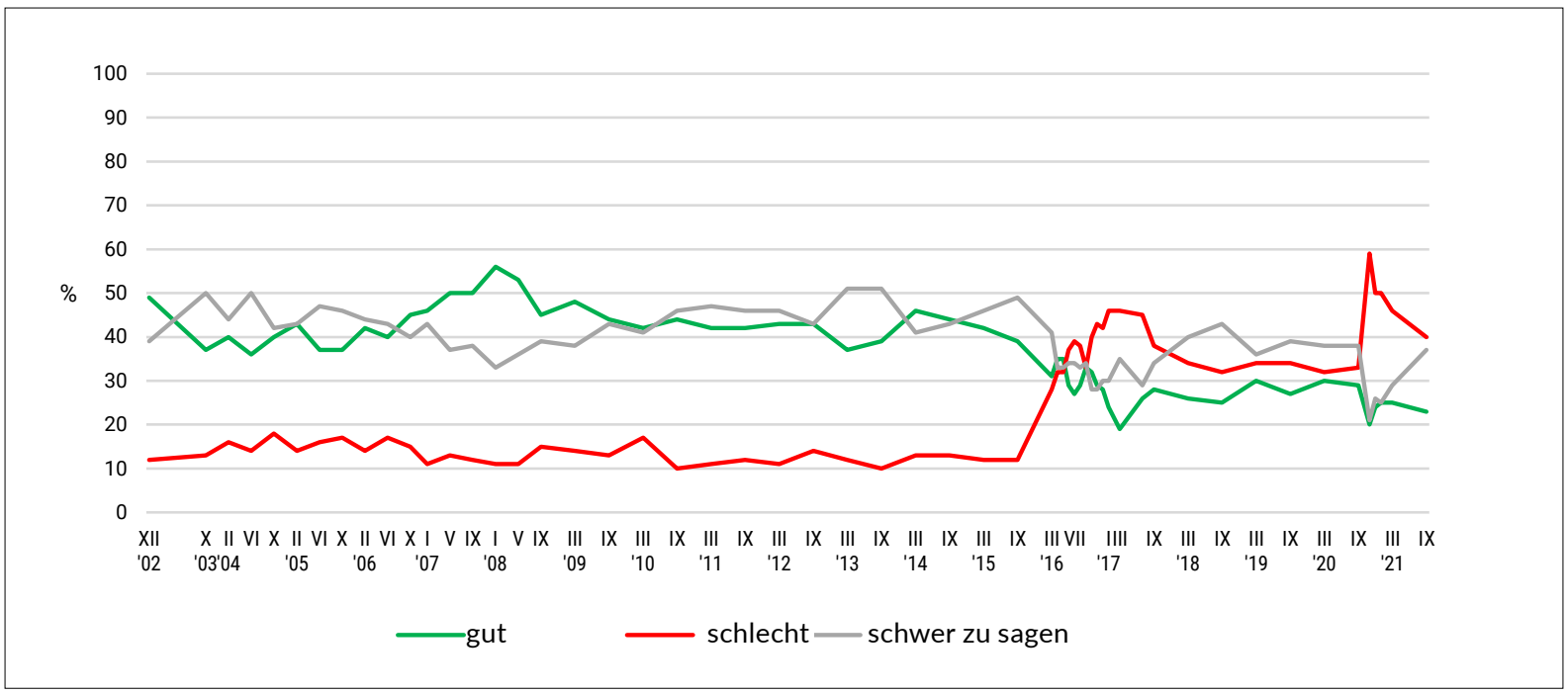

Quelle: CBOS: Komunikat z badań 119/2021: Oceny działalności instytucji publicznych [Bewertungen der Tätigkeiten öffentlicher Institutionen]. Warszawa 10/2021. www.cbos.pl

Grafik 2: Bewertungen der Tätigkeit des Verfassungstribunals (September 2016 - September 2021, \%)

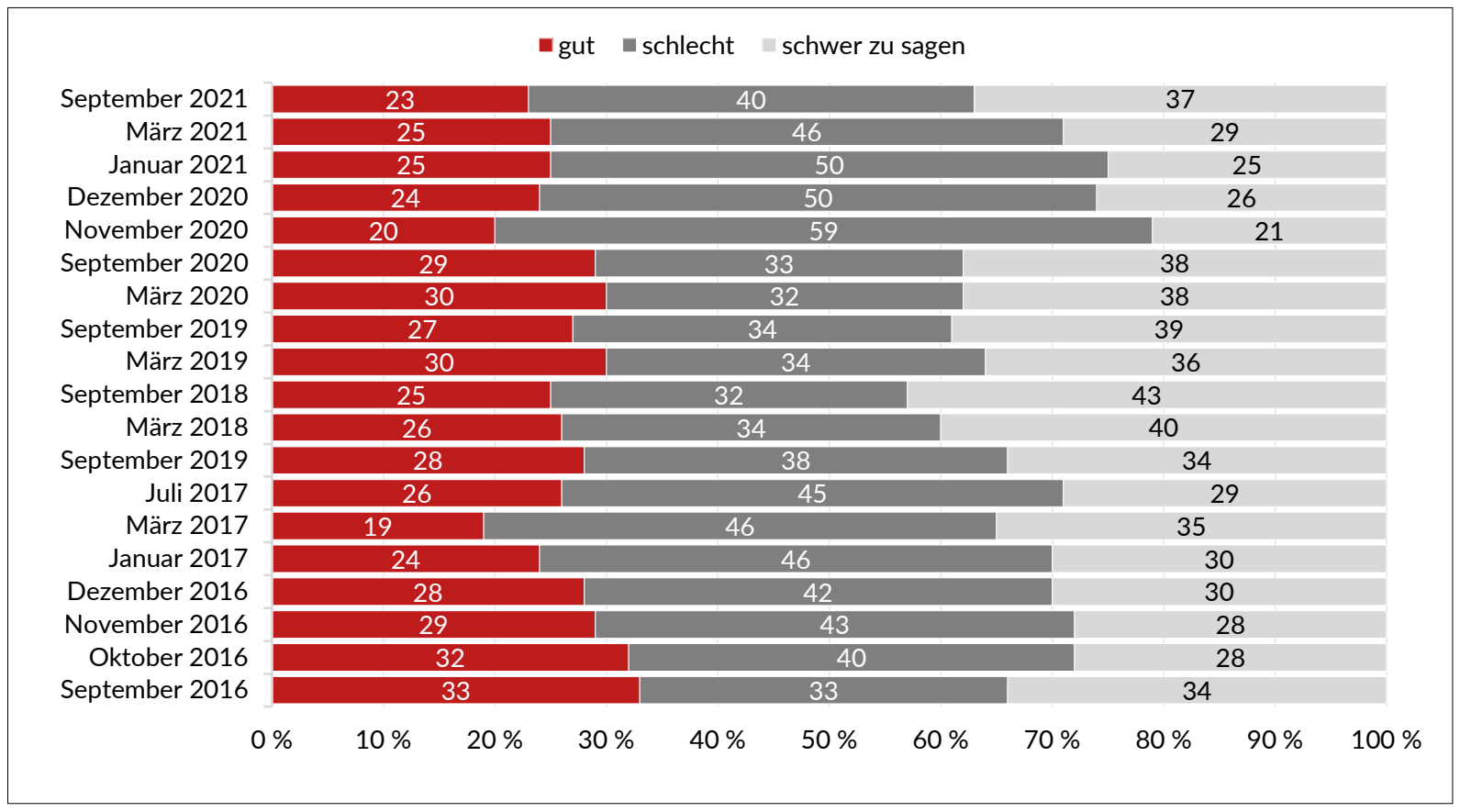

Quelle: CBOS: Komunikat z badań 119/2021: Oceny działalności instytucji publicznych [Bewertungen der Tätigkeiten öffentlicher Institutionen]. Warszawa 10/2021. www.cbos.pl 
Grafik 3: Bewertungen der Tätigkeit des Verfassungstribunals (nach Parteipräferenzen, September 2021 \%)

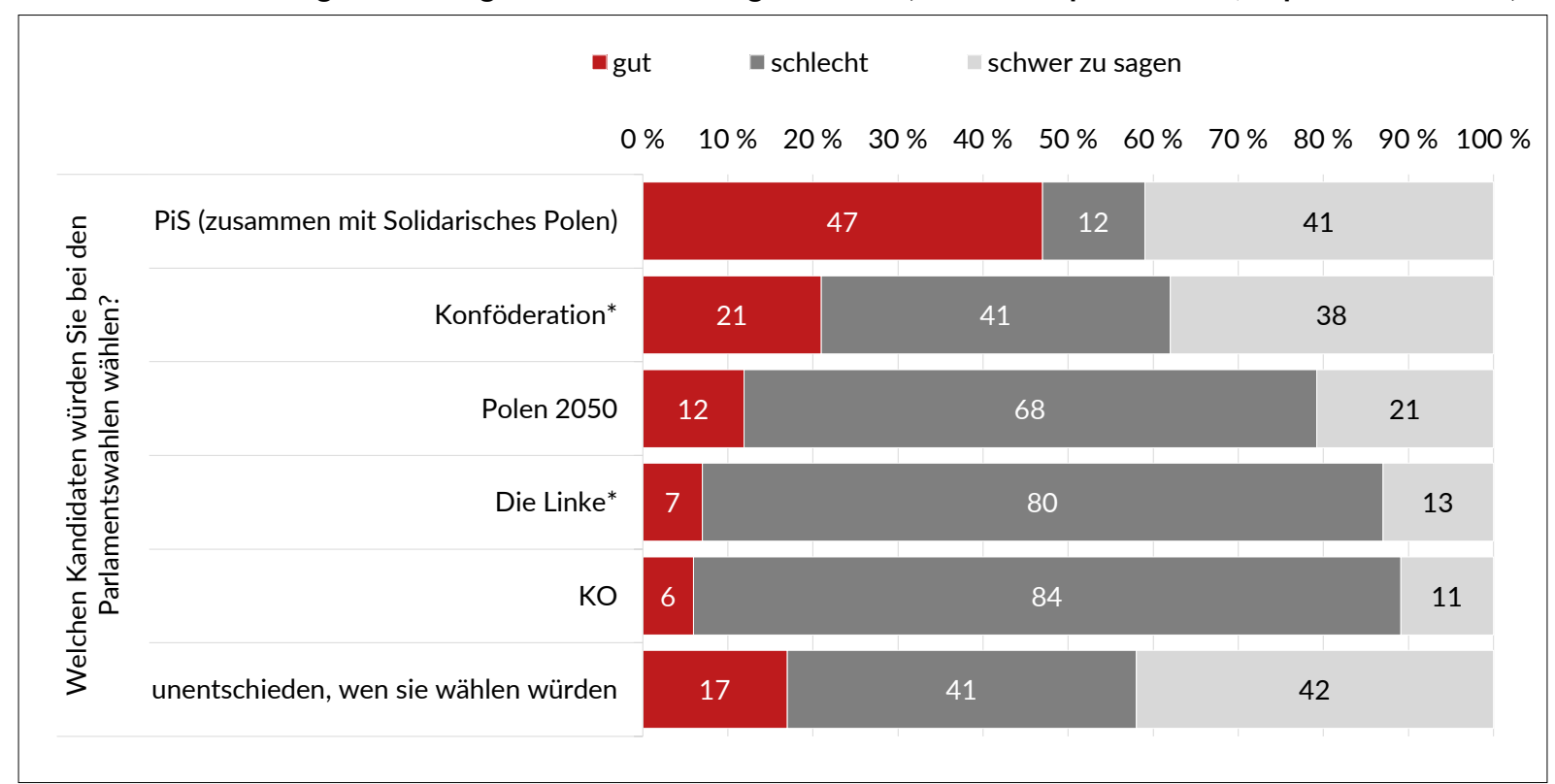

*geringe Anzahl in der Befragung

PiS - Prawo i Sprawiedliwość/Recht und Gerechtigkeit; Solidarisches Polen/Solidarna Polska; Konföderation Freiheit und Unabhängigkeit/Konfederacja Wolność i Niepodległość; Polen 2050/Polska 2050 Szymona Hołownia; Die Linke/Lewica; KO - Koalicja Obywatelska/Bürgerkoalition

Quelle: CBOS: Komunikat z badań 119/2021: Oceny działalności instytucji publicznych [Bewertungen der Tätigkeiten öffentlicher Institutionen]. Warszawa 10/2021. www.cbos.pl

\section{Grafik 4: Bewertungen der Tätigkeit der Gerichte (Dezember 1997 - September 2021, \%)}

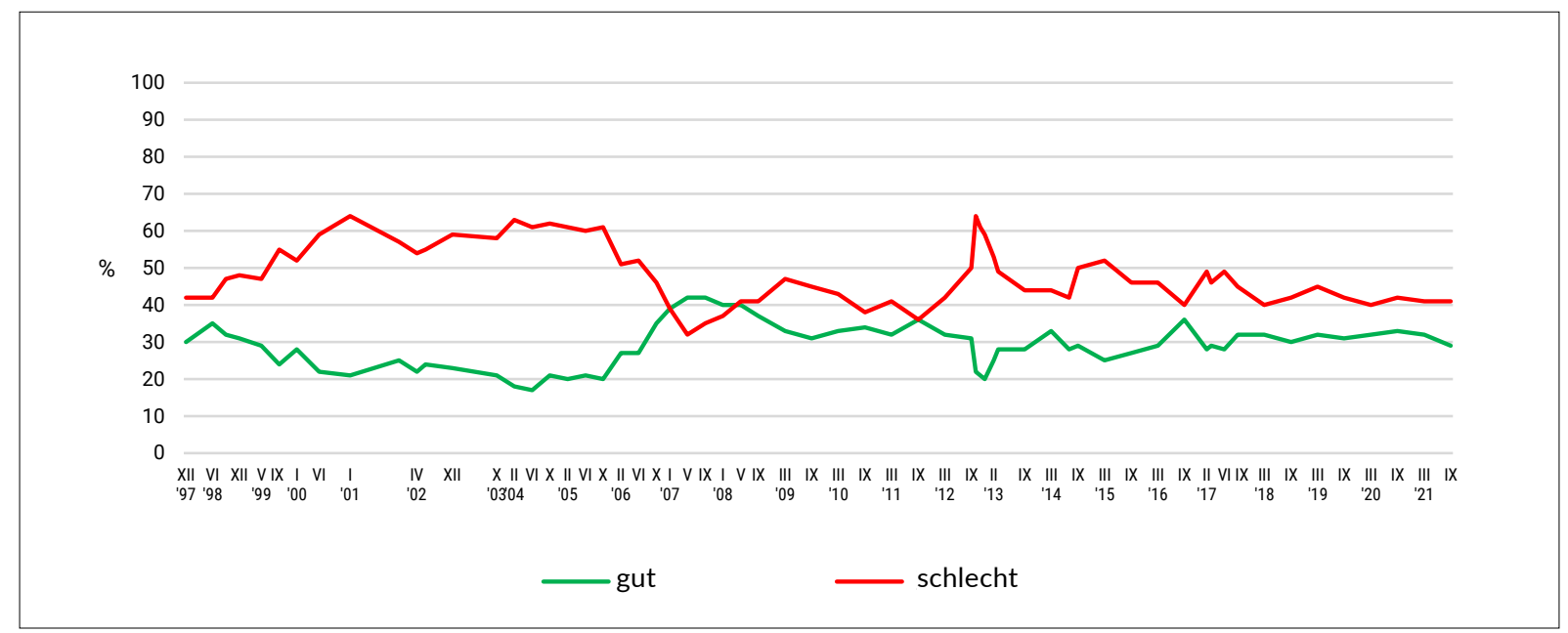

Quelle: CBOS: Komunikat z badań 119/2021: Oceny działalności instytucji publicznych [Bewertungen der Tätigkeiten öffentlicher Institutionen]. Warszawa 10/2021. www.cbos.pl 
Grafik 5: Bewertungen der Tätigkeit der Staatsanwaltschaften (Dezember 1997 - September 2021, \%)

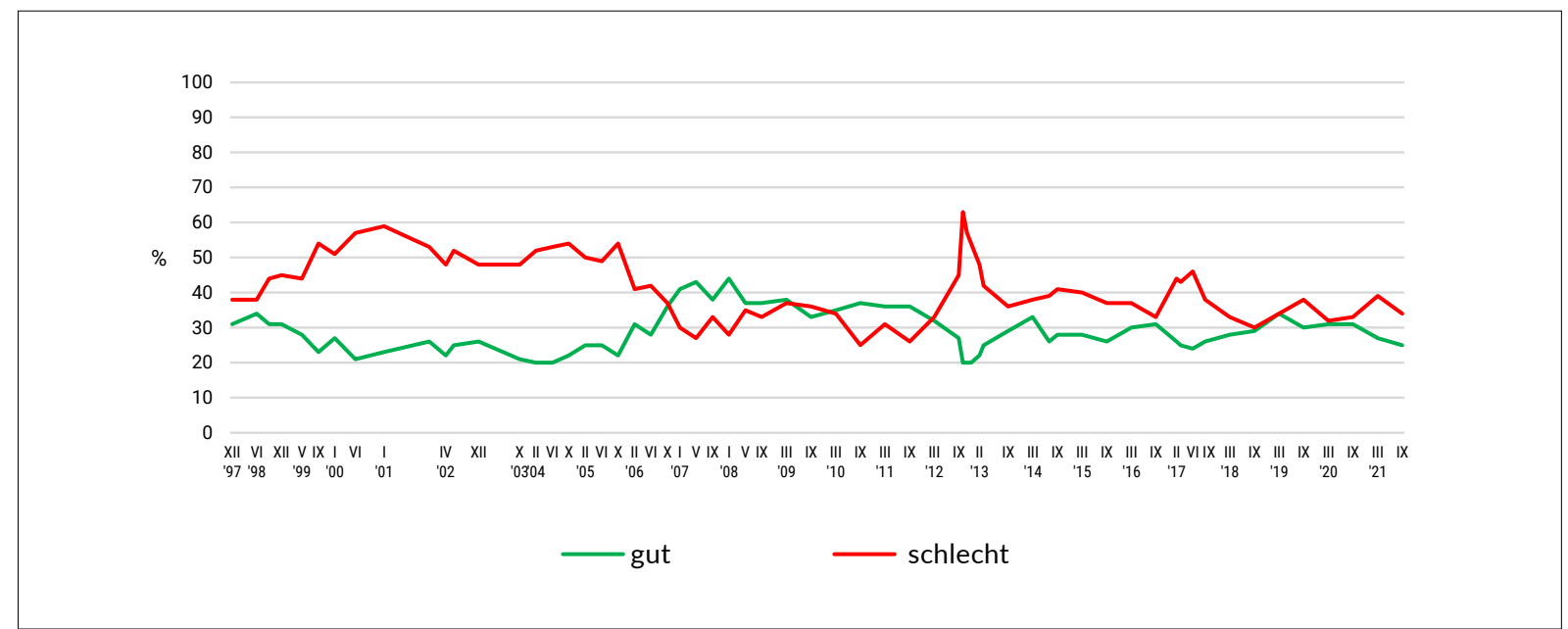

Quelle: CBOS: Komunikat z badań 119/2021: Oceny działalności instytucji publicznych [Bewertungen der Tätigkeiten öffentlicher Institutionen]. Warszawa 10/2021. www.cbos.pl

\section{Grafik 6: Bewertungen der Tätigkeit der Gerichte (März 2015 - September 2021, \%)}

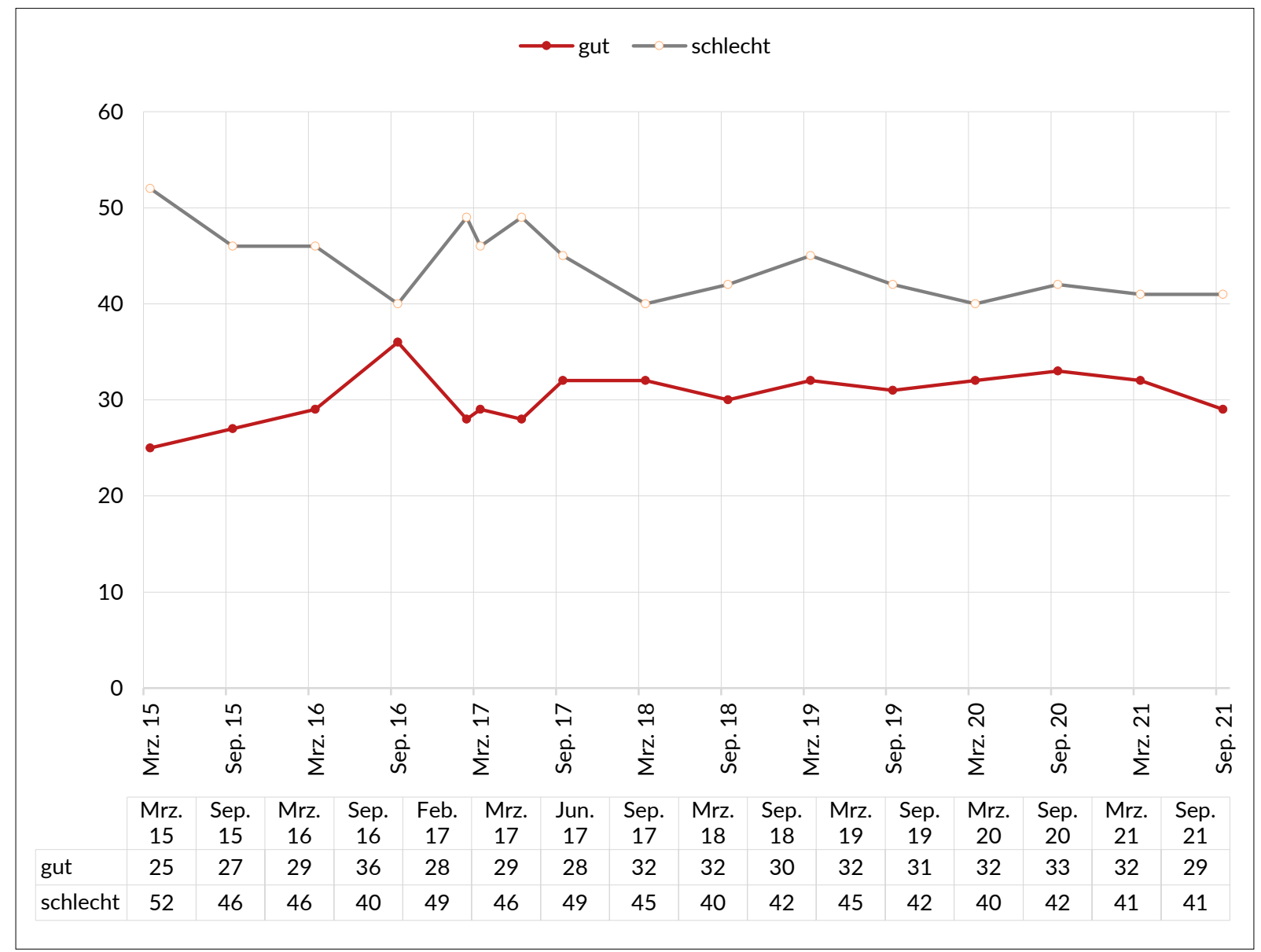

Quelle: CBOS: Komunikat z badań 119/2021: Oceny działalności instytucji publicznych [Bewertungen der Tätigkeiten öffentlicher Institutionen]. Warszawa 10/2021. www.cbos.pl 
Grafik 7: Bewertungen der Tätigkeit der Staatsanwaltschaften (März 2015 - September 2021, \%)

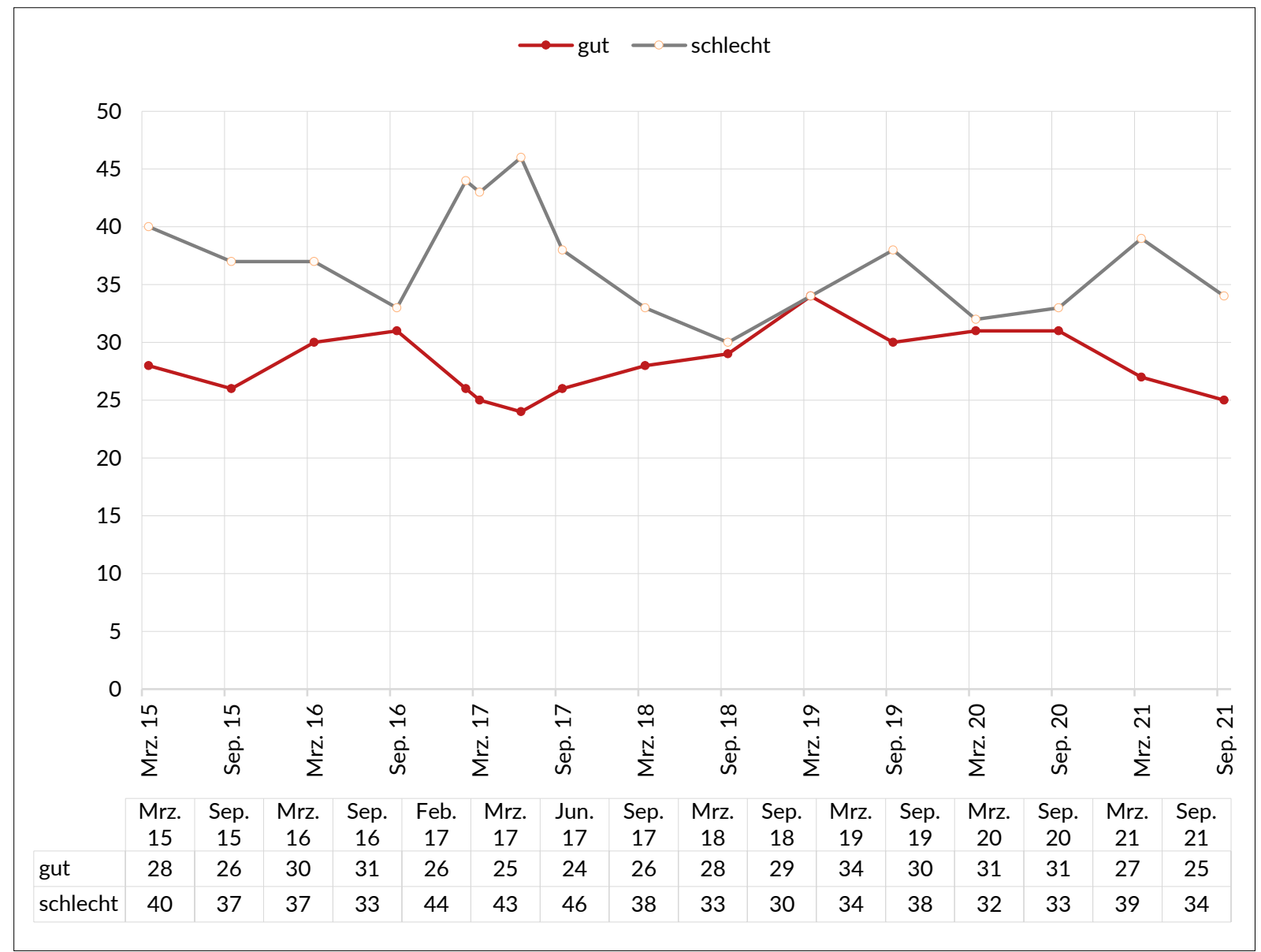

Quelle: CBOS: Komunikat z badań 119/2021: Oceny działalności instytucji publicznych [Bewertungen der Tätigkeiten öffentlicher Institutionen]. Warszawa 10/2021. www.cbos.pl

\section{Einstellungen der polnischen Bevölkerung zur EU}

Grafik 1: Sollten die staatlichen Institutionen in Polen die Empfehlungen und Entscheidungen der EU-Organe zur Justizreform in Polen berücksichtigen? (November 2018 - Oktober 2021, \%)

\begin{tabular}{|c|c|c|c|c|c|c|c|c|c|}
\hline & घauf jeden Fall & eher ja & - eh & nein & I auf kei & Fall & \multicolumn{2}{|c|}{ schwer zu sagen } & \\
\hline Oktober 2021 & 26 & \multicolumn{2}{|c|}{20} & \multicolumn{2}{|r|}{17} & 16 & \multicolumn{3}{|c|}{21} \\
\hline Februar 2020 & 26 & \multicolumn{3}{|c|}{26} & 18 & & 16 & \multicolumn{2}{|c|}{14} \\
\hline November 2018 & 24 & \multicolumn{3}{|c|}{29} & 16 & & 15 & \multicolumn{2}{|l|}{16} \\
\hline & $10 \%$ & $30 \%$ & $40 \%$ & $50 \%$ & $60 \%$ & $70 \%$ & $80 \%$ & $90 \%$ & $100 \%$ \\
\hline
\end{tabular}

Quelle: CBOS: Komunikat z badań 127/2021: Polacy o sporze z UE i członkostwie w tej organizacji [Die Polen über den Streit mit der EZ und der Mitgliedschaft in dieser Organisation]. Warszawa 10/2021. www.cbos.pl 
Grafik 2: $\quad$ Sollten die staatlichen Institutionen in Polen die Empfehlungen und Entscheidungen der EU-Organe zur Justizreform in Polen berücksichtigen? (nach Parteipräferenzen, Oktober 2021, \%)

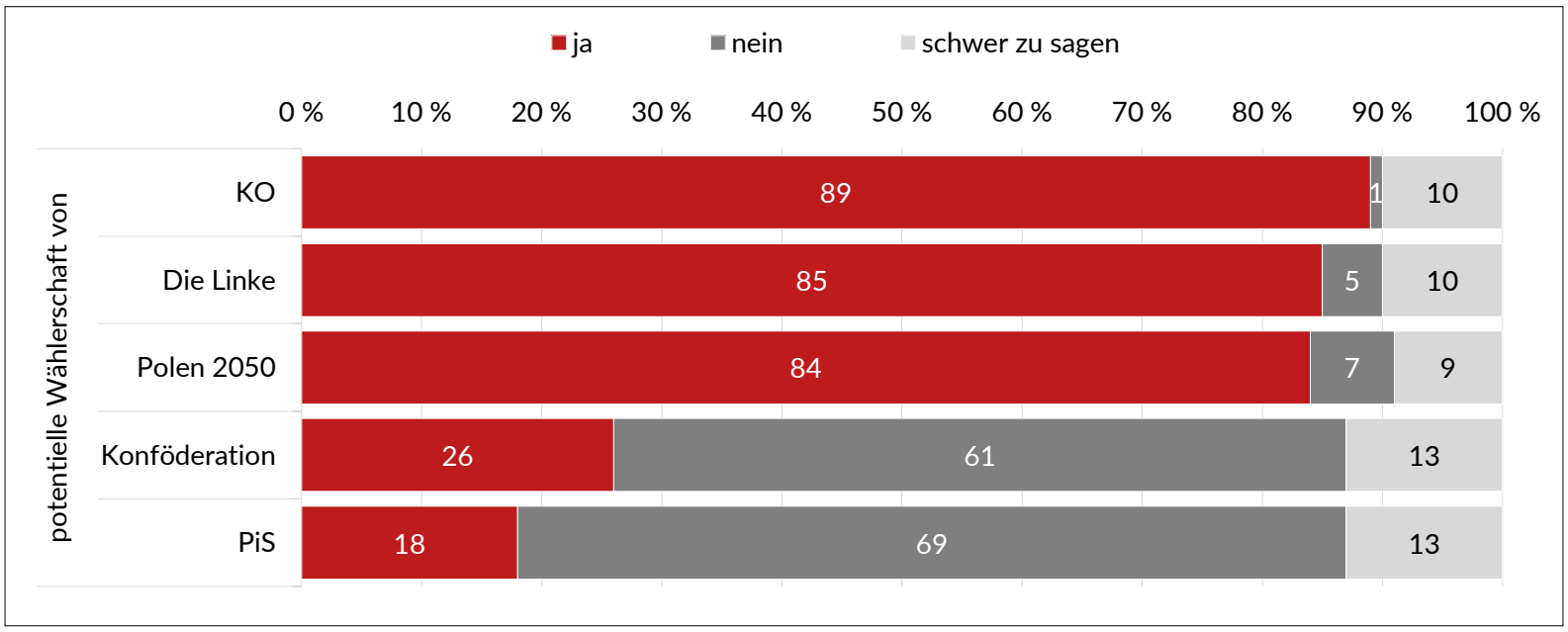

KO - Koalicja Obywatelska/Bürgerkoalition; Die Linke/Lewica (=Neue Linke/Nowa Lewica - früher: SLD - Sojusz Lewicy Demokratycznej/Demokratische Linksallianz, Frühling/Wiosna, Linke Gemeinsam/Lewica Razem); Polen 2050/Polska 2050 Szymona Hołownia; Konföderation Freiheit und Unabhängigkeit/Konfederacja Wolność i Niepodległość (=Konföderation Freiheit und Unabhängigkeit, Nationale Bewegung/Ruch Narodowy, Braun); PiS - Prawo i Sprawiedliwość/Recht und Gerechtigkeit

Quelle: CBOS: Komunikat z badań 127/2021: Polacy o sporze z UE i członkostwie w tej organizacji [Die Polen über den Streit mit der EU und die Mitgliedschaft in dieser Organisation]. Warszawa 10/2021. www.cbos.pl

Grafik 3: Die Einstellung zur Mitgliedschaft Polens in der Europäischen Union (April 2016 - Oktober 2021, \%)

\begin{tabular}{|c|c|c|c|c|c|c|c|c|c|c|}
\hline \multicolumn{11}{|c|}{ - Anhänger $\square$ Gegner $\square$ Unentschiedene } \\
\hline Oktober 2021 & \multicolumn{8}{|c|}{90} & 6 & 4 \\
\hline Dezember 2020 & \multicolumn{8}{|c|}{88} & 6 & 6 \\
\hline Mai/Juni 2020 & \multicolumn{8}{|c|}{87} & 8 & 5 \\
\hline Februar 2020 & \multicolumn{8}{|c|}{89} & 7 & 4 \\
\hline April 2019 & \multicolumn{8}{|c|}{91} & 5 & 4 \\
\hline März 2019 & \multicolumn{8}{|c|}{91} & 5 & 4 \\
\hline Februar 2019 & \multicolumn{8}{|c|}{88} & 8 & 4 \\
\hline November 2018 & \multicolumn{8}{|c|}{87} & 7 & 6 \\
\hline April 2018 & \multicolumn{8}{|c|}{88} & 8 & 4 \\
\hline Januar 2018 & \multicolumn{8}{|c|}{87} & 10 & 3 \\
\hline Dezember 2017 & \multicolumn{8}{|c|}{85} & 8 & 7 \\
\hline Juni 2017 & \multicolumn{8}{|c|}{88} & 9 & 3 \\
\hline April 2017 & \multicolumn{8}{|c|}{88} & 8 & 4 \\
\hline Februar 2017 & \multicolumn{8}{|c|}{85} & 10 & 5 \\
\hline Oktober 2016 & \multicolumn{8}{|c|}{84} & 10 & 6 \\
\hline Juli 2016 & \multicolumn{8}{|c|}{84} & 11 & 5 \\
\hline Juni 2016 & \multicolumn{8}{|c|}{83} & 9 & 8 \\
\hline Mai 2016 & \multicolumn{8}{|c|}{83} & 9 & 8 \\
\hline April 2016 & \multicolumn{8}{|c|}{85} & 9 & 6 \\
\hline 09 & $10 \%$ & $20 \%$ & $30 \%$ & $40 \%$ & $50 \%$ & $60 \%$ & $70 \%$ & $80 \%$ & $90 \%$ & $100 \%$ \\
\hline
\end{tabular}

Quelle: CBOS: Komunikat z badań 127/2021: Polacy o sporze z UE i członkostwie w tej organizacji [Die Polen über den Streit mit der EU und die Mitgliedschaft in dieser Organisation]. Warszawa 10/2021. www.cbos.pl 
Grafik 4: Die Einstellung zur Mitgliedschaft Polens in der Europäischen Union (1994 - Oktober 2021, \%)

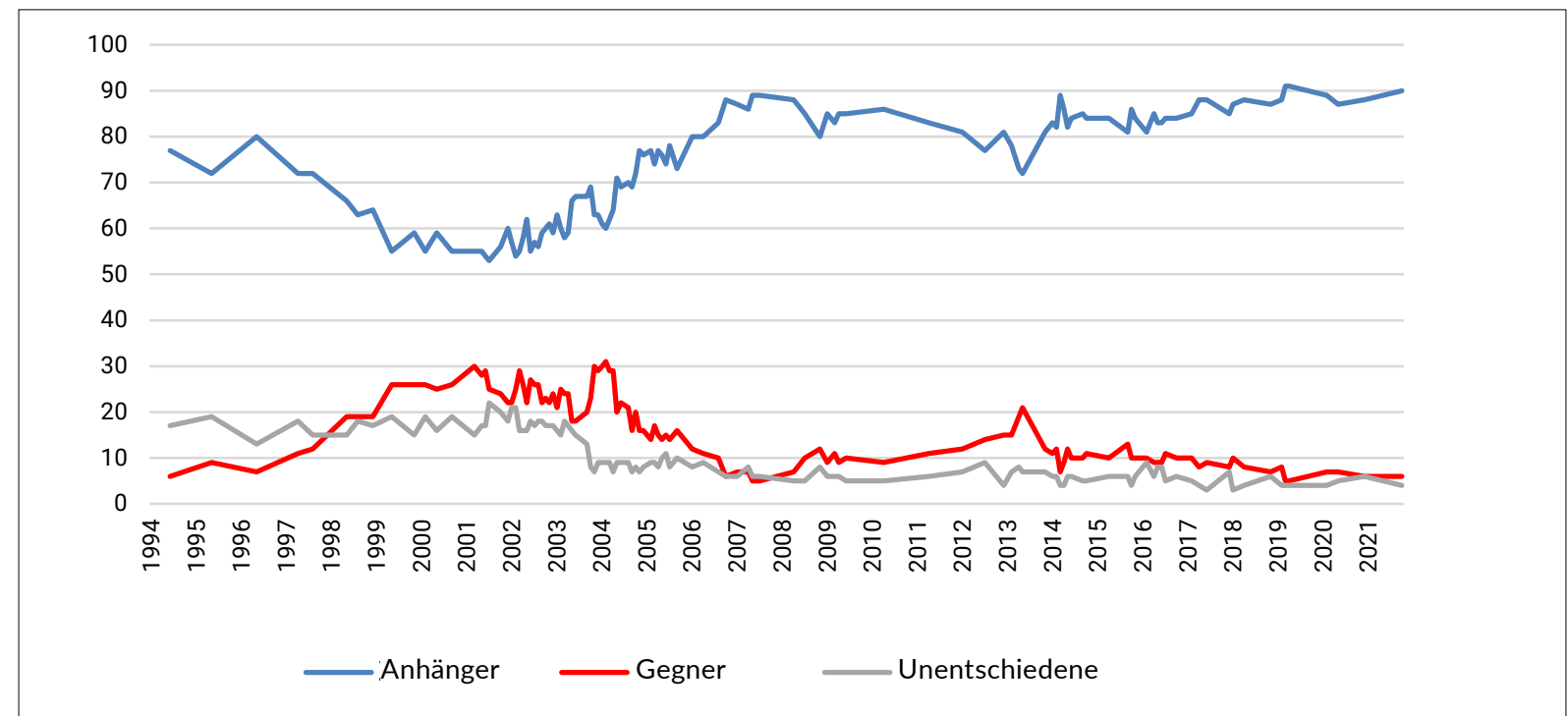

Quelle: CBOS: Komunikat z badań 127/2021: Polacy o sporze z UE i członkostwie w tej organizacji [Die Polen über den Streit mit der EU und die Mitgliedschaft in dieser Organisation]. Warszawa 10/2021. www.cbos.pl

\section{Oktober - 1. November 2021}

\begin{tabular}{|c|c|}
\hline 19.10 .2021 & $\begin{array}{l}\text { Die Präsidentin der Europäischen Kommission, Ursula von der Leyen, und der Ministerpräsident Polens, Mateusz } \\
\text { Morawiecki, nehmen an der Debatte im Europäischen Parlament zur "Krise der Rechtsstaatlichkeit in Polen } \\
\text { und dem Vorrang von EU-Recht« teil. Hintergrund ist das Urteil des polnischen Verfassungstribunals (Try- } \\
\text { bunał Konstytucyjny - TK ) vom 7. Oktober, dass das EU-Recht nur »in gewissen Grenzen« über polnischem } \\
\text { Recht steht. Die EU wertet das als Verletzung der europäischen Verträge und Werte. Von der Leyen hebt die } \\
\text { Rechtsstaatlichkeit als gemeinsame Grundlage der Europäischen Union hervor. Die Europäische Kommission } \\
\text { werde handeln; Maßnahmen wären eine Klage vor dem Europäischen Gerichtshof, ein Rechtsstaatsverfahren, } \\
\text { die Zurückhaltung finanzieller Mittel oder die Anwendung des Anfang des Jahres beschlossenen "EU-Rechts- } \\
\text { staatsmechanismus«. Morawiecki sagt in seiner zeitlich deutlich überzogenen Rede, dass sich Polen nicht von } \\
\text { der EU erpressen lasse. Er zeigt sich besorgt, dass sich die EU zu einem bürokratischen Superstaat entwickele, } \\
\text { der sich zunehmend in die Belange der Mitgliedsstaaten einmischt. }\end{array}$ \\
\hline 20.10 & $\begin{array}{l}\text { Die Regierung der Bundesrepublik Deutschland bietet Polen an, die Präsenz der Bundespolizei im deutsch-polnischen } \\
\text { Grenzgebiet zu erhöhen. Bundesinnenminister Horst Seehofer sagt, man unterstütze Polen bei der Abwehr illegaler } \\
\text { Einreise, verfolge aber keine Schließung der deutsch-polnischen Grenze. Hintergrund sind die illegalen Grenzüber- } \\
\text { tritte an der polnisch-belarussischen Grenze von Migranten aus afrikanischen und asiatischen Ländern, die der bela- } \\
\text { russische Staatspräsident Alexander Lukaschenko zielgerichtet herbeigeführt hat. Ein Teil der Migranten zieht weiter } \\
\text { in Richtung Deutschland, wo im Oktober 3.200 Personen mit einem Bezug zur Belarus-Route registriert wurden. }\end{array}$ \\
\hline 21.10 .2021 & $\begin{array}{l}\text { Das Europaparlament verabschiedet eine Resolution (502 Ja-Stimmen, } 153 \text { Nein-Stimmen, } 16 \text { Enthaltun- } \\
\text { gen), in der es das Urteil des polnischen Verfassungstribunals (Trybunał Konstytucyjny - TK ) vom 7. Okto- } \\
\text { ber } 2021 \text { als »Angriff auf die europäische Wertegemeinschaft und das Recht als Ganzes« verurteilt. Gefor- } \\
\text { dert wird, Finanzmittel der Europäischen Union keinen Regierungen zur Verfügung zu stellen, die absichtlich } \\
\text { und systematisch die europäischen Werte missachten. Die Europäische Kommission und der Europarat wer- } \\
\text { den aufgefordert, entsprechende Maßnahmen zu ergreifen. Die Resolution hat keine bindende Wirkung. Das } \\
\text { Urteil des TK kam zu dem Schluss, dass Teile der Bestimmungen der europäischen Verträge nicht mit der pol- } \\
\text { nischen Verfassung vereinbar sind. }\end{array}$ \\
\hline
\end{tabular}




\begin{tabular}{|c|c|}
\hline 23.10 .2021 & $\begin{array}{l}\text { Unter dem Motto "Mütter an die Grenze. Kinder gehören nicht in den Wald« findet in Michałowo an der pol- } \\
\text { nisch-belarussischen Grenze eine Demonstration für die Zulassung medizinischer und humanitärer Organi- } \\
\text { sationen in die mit Ausnahmezustands belegte Zone statt. Unterstützt wird die Aktion von den Ehefrauen } \\
\text { ehemaliger Präsidenten, Danuta Wałesa, Jolanta Kwaśniewska und Anna Komorowska. Im Grenzgebiet hal- } \\
\text { ten sich seit Wochen Migranten aus dem Nahen Osten und anderen Ländern auf, die der belarussische Staats- } \\
\text { präsident Alexander Lukaschenko gezielt dorthin geholt hat. Polen will sie nicht aufnehmen, Belarus lässt sie } \\
\text { nicht ins Land zurück. }\end{array}$ \\
\hline 23.10 .2021 & $\begin{array}{l}\text { Donald Tusk wird in einer allgemeinen Mitgliederabstimmung mit } 97,4 \% \text { der Stimmen als Parteichef der } \\
\text { Bürgerplattform (Platforma Obywatelska) bestätigt. Außerdem wurden die PO-Vorsitzenden auf Woiwod- } \\
\text { schafts- und Kreisebene gewählt. }\end{array}$ \\
\hline 25.10 .2021 & $\begin{array}{l}\text { Auf Antrag des Landesjustizrates (Krajowa Rada Sądownictwa - KRS) beruft Präsident Andrzej Duda } 100 \\
\text { neue Richter in verschiedene Gerichten auf Woiwodschaftsebene sowie darunter liegenden Ebenen. Der KRS } \\
\text { ist ein rechtlich umstrittenes Gremium, dem vorgeworfen wird, von der Regierungskoalition politisch instru- } \\
\text { mentalisiert zu werden. }\end{array}$ \\
\hline 26.10 .2021 & $\begin{array}{l}\text { Infolge einer Regierungsumbildung vereidigt Präsident Andrzej Duda Henryk Kowalczyk als Minister für Land- } \\
\text { wirtschaft und Entwicklung des ländlichen Raumes sowie Vizeministerpräsident, Piotr Nowak als Minister für } \\
\text { Entwicklung und Technologie, Tadeusz Kościński als Finanzminister, Grzegorz Puda als Minister für Fonds } \\
\text { und Regionalpolitik, Anna Moskwa als Ministerin für Klima und Umwelt und Kamil Bortniczuk als Minis- } \\
\text { ter für Sport und Tourismus. Der Umbildung ging voraus, dass Jarosław Gowin, Parteichef von Verständi- } \\
\text { gung (Porozumienie), als Minister für Entwicklung, Arbeit und Technologie im August entlassen wurde und } \\
\text { die Regierungskoalition mit einigen Abgeordneten verlassen hat. Andere Vertreter von Verständigung wech- } \\
\text { selten zur größten Partei der Regierungskoalition, Recht und Gerechtigkeit (Prawo i Sprawiedliwość-PiS). }\end{array}$ \\
\hline 27.10 .2021 & $\begin{array}{l}\text { Der Gerichtshof der Europäischen Union (EuGH) in Luxemburg verhängt gegen Polen Strafzahlungen an die } \\
\text { Europäische Kommission in Höhe von einer Million Euro pro Tag, da Polen die Entscheidung des EuGH vom } \\
\text { Juli, die Arbeit der Disziplinarkammer (Izba Dyscyplinarna) für Richter auszusetzen, nicht umgesetzt hat. Das } \\
\text { Zwangsgeld muss so lange gezahlt werden, bis Polen der Entscheidung nachkommt oder bis der EuGH das end- } \\
\text { gültige Urteil in dieser Angelegenheit fällt. Die Disziplinarkammer am Obersten Gericht (Sąd Najwyższy), die } \\
\text { die Regierung von Recht und Gerechtigkeit (Prawo i Sprawiedliwość - PiS) im Rahmen ihres Justizumbaus } \\
\text { eingerichtet hat, kann Richter bestrafen und entlassen. Der EuGH vertritt den Standpunkt, dass die Kammer } \\
\text { nicht mit EU-Recht vereinbar ist. }\end{array}$ \\
\hline 28.10 .2021 & $\begin{array}{l}\text { Justizminister Zbigniew Ziobro sagt mit Blick auf die Verfügung des Gerichtshofes der Europäischen Union } \\
\text { (EuGH) in Luxemburg vom Vortag, Polen solle die verhängte Strafe an die Europäische Kommission nicht } \\
\text { zahlen. Der Hintergrund für das Zwangsgeld ist, dass der EuGH im Juli entschieden hat, dass die Disziplinar- } \\
\text { kammer (Izba Dyscyplinarna) am Obersten Gericht (Sąd Najwyższy) ihre Arbeit einstellen muss, da sie gegen } \\
\text { EU-Recht verstoße. Polen hat die Entscheidung nicht umgesetzt. }\end{array}$ \\
\hline 29.10 .2021 & $\begin{array}{l}\text { Das Europaparlament reicht Klage gegen die Europäische Kommission beim Gerichtshof der Europäischen } \\
\text { Union (EuGH) ein, weil diese den EU-Rechtsstaatsmechanismus bislang nicht angewendet hat. Dieser sieht } \\
\text { vor, dass EU-Mitgliedstaaten Gelder gekürzt werden können, wenn ein Missbrauch des Geldes wegen Rechts- } \\
\text { staatsverstößen droht. Der Kontext ist, dass Kritiker der polnischen Regierung vorwerfen, dass sie die Justiz- } \\
\text { behörden in Polen für politische Zwecke instrumentalisiert. Eine Gefahr für den EU-Haushalt sehen die Kriti- } \\
\text { ker, da die nationale Justiz für die Aufklärung bei möglichem Missbrauch von EU-Finanzmitteln zuständig ist. }\end{array}$ \\
\hline 31.10 .2021 & $\begin{array}{l}\text { Laut einer Verordnung des Ministerrates, die heute in Kraft tritt, werden die aktuell geltenden Bestimmungen } \\
\text { zur Eindämmung der herrschenden Corona-Pandemie bis zum 30. November } 2021 \text { verlängert. Das bedeutet } \\
\text { u. a. das Einhalten eines Mindestabstands von 1,5 m für Personen, die nicht im selben Haushalt leben, und das } \\
\text { Tragen einer Mund-Nase-Bedeckung im öffentlichen Personenverkehr, Geschäften, Behörden, Kirchen sowie } \\
\text { in Schulen und Universitäten während der Pausen. In Veranstaltungsräumen (Theater, Kino) gilt weiter eine } \\
\text { Beschränkung der Besucherzahl. }\end{array}$ \\
\hline 01.11 .2021 & $\begin{array}{l}\text { Nach aktuellen Angaben des Gesundheitsministeriums wurden in Polen } 3.030151 \text { Covid-19-Infektionen seit } \\
\text { Dezember } 2019 \text { festgestellt. } 77.012 \text { Personen sind im Zusammenhang mit dem Corona-Virus gestorben. }\end{array}$ \\
\hline
\end{tabular}

Sie können die gesamte Chronik seit 2007 auch auf http://www.laender-analysen.de/polen/ unter dem Link "Chronik« lesen. 
Die Polen-Analysen erscheinen zweimal monatlich als E-Mail-Dienst. Sie werden gemeinsam vom Deutschen PolenInstitut Darmstadt, der Forschungsstelle Osteuropa an der Universität Bremen und weiteren Partnern eines Konsortiums (siehe Titelseite) herausgegeben.

Ein Archiv der Polen-Analysen finden Sie im Internet unter www.laender-analysen.de/polen Kostenloses Abonnement unter http://www.deutsches-polen-institut.de/Newsletter/subscribe.php

Diese Analysen finden Sie online als Lizenzausgabe auf bpb.de

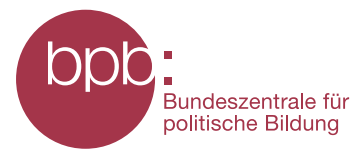

Deutsches Polen-Institut Darmstadt (www.deutsches-polen-institut.de)

Das seit 1980 tätige Deutsche Polen-Institut Darmstadt (DPI) ist ein Forschungs-, Informations- und Veranstaltungszentrum für polnische Kultur, Geschichte, Politik, Gesellschaft und die deutsch-polnischen Beziehungen, die sich im Kontext der europäischen Integration entwickeln. Institutionelle Träger des DPI sind das Land Hessen, die Kultusminister der Länder, das Auswärtige Amt und die Wissenschaftsstadt Darmstadt. Einen wesentlichen Beitrag zur Verwirklichung der Institutsziele leisten private Stiftungen. Ziel der Vermittlertätigkeit des DPI ist es, "die zu interessieren, auf die es politisch, wirtschaftlich, gesellschaftlich und kulturell im deutsch-polnischen Verhältnis ankommt (Leitlinien 1997). Es geht um die Entscheider und Multiplikatoren in Politik, Kultur, Bildung, Verwaltung, Medien und Wirtschaft. Das DPI versteht sich in Kooperation mit den Orten wissenschaftlicher Polen-Kompetenz an deutschen Hochschulen und Forschungsinstituten als verbindendes und vernetzendes Zentrum. Mit der 70.000 Bände zählenden multidisziplinären Fachbibliothek für Polen, die eine einzigartige Sammlung polnischer Belletristik in der Originalsprache und in deutscher Übersetzung umfasst, ist das DPI ein geschätzter Ort der Recherche und des wissenschaftlichen Arbeitens.

Forschungsstelle Osteuropa an der Universität Bremen (www.forschungsstelle.uni-bremen.de)

1982 gegründet, widmet sich die Forschungsstelle Osteuropa an der Universität Bremen der interdisziplinären Analyse der Länder Ost- und Ostmitteleuropas in Zeitgeschichte und Gegenwart. Der Forschungsschwerpunkt liegt dabei auf der Rolle von "Dissens und Konsens«, von Opposition und Zivilgesellschaft in ihrem historischen, politischen, gesellschaftlichen und kulturellen Kontext. Die Forschungsstelle besitzt in ihrem Archiv eine einzigartige Sammlung alternativer Kulturgüter und unabhängiger Texte aus den ehemaligen sozialistischen Ländern. Darunter befindet sich auch eine umfangreiche Sammlung des "Zweiten Umlaufs«, die das Schrifttum und Dokumente unabhängiger Initiativen und gesellschaftlicher Gruppen in Polen aus der Zeit von 1976 bis zum Umbruch umfasst. Hinzu kommt eine umfangreiche Bibliothek mit wissenschaftlicher Literatur. Mit Archiv, Bibliothek und zwei wissenschaftlichen Abteilungen ist die Forschungsstelle auch eine Anlaufstelle sowohl für Gastwissenschaftler als auch für die interessierte Öffentlichkeit.

Eine der Hauptaufgaben der Forschungsstelle ist die Information der interessierten Öffentlichkeit. Dazu gehören unter anderem regelmäßige E-Mail-Informationsdienste für Politik, Wirtschaft, Zivilgesellschaft und Medien.

Herausgeber:

Deutsches Polen-Institut, Deutsche Gesellschaft für Osteuropakunde e.V., Forschungsstelle Osteuropa an der Universität Bremen, Leibniz-Institut für Agrarentwicklung in Transformationsökonomien, Leibniz-Institut für Ost- und Südosteuropaforschung, Zentrum für Osteuropa- und internationale Studien (Z0iS) gGmbH

Redaktion:

Dr. Andrzej Kaluza (verantwortlich) (Darmstadt) und Dr. Silke Plate (Bremen)

Satz: Matthias Neumann

Wissenschaftlicher Beirat:

Prof. Dr. Stefan Garsztecki, Technische Universität Chemnitz

Prof. Dr. Klaus Ziemer, Kardinal-Stefan-Wyszyński-Universität Warschau

Die Meinungen, die in den Polen-Analysen geäußert werden, geben ausschließlich die Auffassung der Autoren wieder Abdruck und sonstige publizistische Nutzung sind nach Rücksprache mit der Redaktion gestattet. Polen-Analysen-Layout: Cengiz Kibaroglu, Matthias Neumann

Alle Ausgaben der Polen-Analysen sind mit Themen- und Autorenindex archiviert unter www.laender-analysen.de

ISSN 1863-9712 @ 2021 by Deutsches Polen-Institut, Deutsche Gesellschaft für Osteuropakunde e.V., Forschungsstelle Osteuropa an der Universität Bremen, Leibniz-Institut für Agrarentwicklung in Transformationsökonomien, Leibniz-Institut für Ost- und Südosteuropaforschung, Zentrum für Osteuropa- und internationale Studien (ZOiS) gGmbH Kontakt: Dr. Andrzej Kaluza, Deutsches Polen-Institut, Residenzschloss, Marktplatz 15, 64283 Darmstadt,

Tel.: +49/6151/4202-20, Fax: +49/6151/4202-10, E-Mail: kaluza@dpi-da.de, Internet: www.laender-analysen.de/polen 


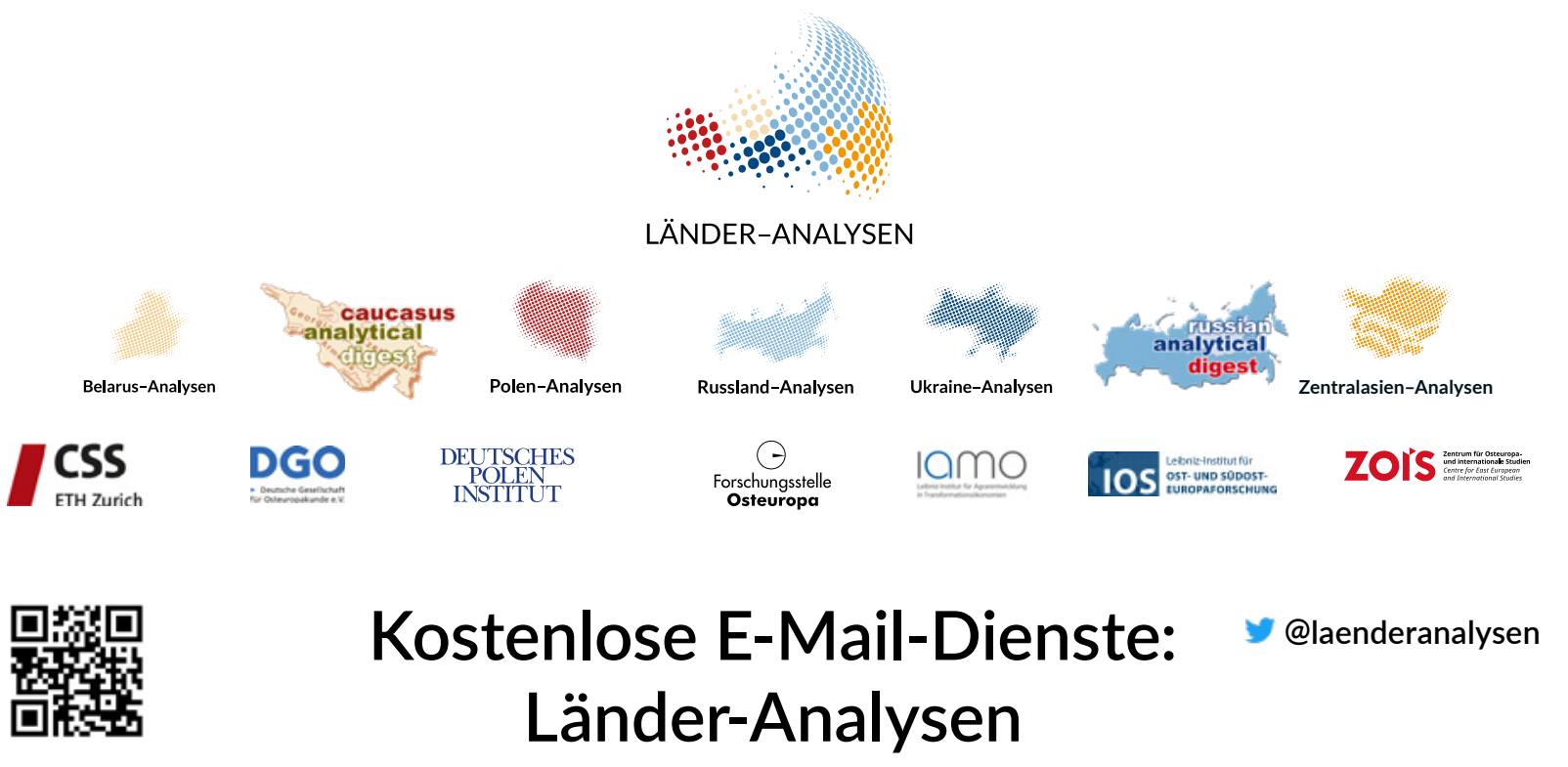

Die Länder-Analysen bieten regelmäßig im kostenlosen Abonnement kompetente Einschätzungen aktueller politischer, wirtschaftlicher, sozialer und kultureller Entwicklungen in Ostmitteleuropa und der GUS. Alle Länder-Analysen verstehen sich als Teil eines gemeinsamen Projektes, das der wissenschaftlich fundierten, allgemeinverständlich formulierten Analyse der Entwicklungen im östlichen Europa, der Offenheit für verschiedene inhaltliche Positionen und der kostenlosen und nicht-kommerziellen Information einer breit verstandenen interessierten Öffentlichkeit verpflichtet ist. Autor/innen sind internationale Fachwissenschaftler/innen und Expert/innen. Die Redaktionen der Länder-Analysen bestehen aus Wissenschaftler/innen mit langjähriger Forschungserfahrung.

Die deutschsprachigen Länder-Analysen werden gemeinsam von der Forschungsstelle Osteuropa an der Universität Bremen, dem Zentrum für Osteuropa- und internationale Studien, der Deutschen Gesellschaft für Osteuropakunde, dem Deutschen Polen-Institut, dem Leibniz-Institut für Agrarentwicklung in Transformationsökonomien und dem Leibniz-Institut für Ost- und Südosteuropaforschung herausgegeben. Die englischsprachigen Länder-Analysen erscheinen in Kooperation der Forschungsstelle Osteuropa mit dem Center for Security Studies (CSS) der ETH Zürich.

Die Länder-Analysen bieten regelmäßig Kurzanalysen zu aktuellen Themen, ergänzt um Grafiken und Tabellen sowie Dokumentationen. Zusätzlich gibt es eine Chronik aktueller Ereignisse.

\section{Belarus-Analysen}

Erscheinungsweise: zweimonatlich

Abonnement unter: http://www.laender-analysen.de/belarus/

\section{Caucasus Analytical Digest}

In englischer Sprache. Erscheinungsweise: zweimonatlich

Abonnement unter: http://www.css.ethz.ch/en/publications/cad.html

\section{Polen-Analysen}

Erscheinungsweise: zweimal monatlich

Abonnement unter: http://www.deutsches-polen-institut.de/newsletter/polen-analysen/

\section{Russland-Analysen}

Erscheinungsweise: zweimal monatlich

Abonnement unter: http://www.laender-analysen.de/russland/

\section{Russian Analytical Digest}

In englischer Sprache. Erscheinungsweise: zweimal monatlich Abonnement unter: http://www.css.ethz.ch/en/publications/rad.html

\section{Ukraine-Analysen}

Erscheinungsweise: zweimal monatlich

Abonnement unter: http://www.laender-analysen.de/ukraine/

\section{Zentralasien-Analysen}

Erscheinungsweise: zweimonatlich

Abonnement unter: http://www.laender-analysen.de/zentralasien/ 